\title{
Lusioersily
}

\section{Segmentation of breast MR images using a generalised 2D mathematical model with inflation and deflation forces of active contours}

Rampun, A., Scotney, B., Morrow, PJ., Wang, H., \& Winder, J. (2019). Segmentation of breast MR images using a generalised 2D mathematical model with inflation and deflation forces of active contours. Artificial Intelligence in Medicine, 97(n/a), 44-60. https://doi.org/10.1016/j.artmed.2018.10.007

Link to publication record in Ulster University Research Portal

\section{Published in:}

Artificial Intelligence in Medicine

Publication Status:

Published (in print/issue): 30/06/2019

DOI:

10.1016/j.artmed.2018.10.007

\section{Document Version}

Author Accepted version

\section{General rights}

Copyright for the publications made accessible via Ulster University's Research Portal is retained by the author(s) and / or other copyright owners and it is a condition of accessing these publications that users recognise and abide by the legal requirements associated with these rights.

\section{Take down policy}

The Research Portal is Ulster University's institutional repository that provides access to Ulster's research outputs. Every effort has been made to ensure that content in the Research Portal does not infringe any person's rights, or applicable UK laws. If you discover content in the Research Portal that you believe breaches copyright or violates any law, please contact pure-support@ulster.ac.uk. 


\title{
Segmentation of Breast MR Images using a Generalised 2D Mathematical Model with Inflation and Deflation Forces of Active Contours
}

\author{
Andrik Rampun, Bryan W. Scotney, Philip J. Morrow \\ School of Computing and Information Engineering, Ulster University, Coleraine, \\ Northern Ireland, BT52 1SA \\ Hui Wang \\ School of Computing and Mathematics, Ulster University, Jordanstown, Newtownabbey, \\ Northern Ireland, BT37 OQB \\ John Winder \\ School of Health Sciences, Ulster University, Newtownabbey, Northern Ireland, BT37 0QB
}

\begin{abstract}
In medical computer aided diagnosis systems, image segmentation is one of the major pre-processing steps used to ensure only the region of interest, such as the breast region, will be processed in subsequent steps. Nevertheless, breast segmentation is a difficult task due to low contrast and inhomogeneity, especially when estimating the chest wall in magnetic resonance (MR) images. In fact, the chest wall is comprises of fat, skin, muscles, and the thoracic skeleton, which can misguide automatic methods when attempting to estimate its location. The objective of the study is to develop a fully automated method for breast and pectoral muscle boundary estimation in MR images. Firstly, we develop a 2D breast mathematical model based on $30 \mathrm{MRI}$ slices (from a patient) and identify important landmarks to obtain a model for the general shape of the breast in an axial plane. Subsequently, we use Otsu's thresholding approach and Canny
\end{abstract}

\footnotetext{
${ }^{\text {th }}$ Fully documented templates are available in the elsarticle package on CTAN

${ }^{*}$ Corresponding authors: A. Rampun, P. J. Morrow and B. W. Scotney

Email address: y.rampun, pj.morrow, bw.scotney@ulster.ac.uk (Andrik Rampun, Bryan W. Scotney, Philip J. Morrow)
}

Preprint submitted to Journal of Artificial Intelligence in Medicine 
edge detection to estimate the breast boundary. The active contour method is then employed using both inflation and deflation forces to estimate the pectoral muscle boundary by taking account of information obtained from the proposed 2D model. Finally, the estimated boundary is smoothed using a median filter to remove outliers. Our two datasets contain 60 patients in total and the proposed method is evaluated based on 59 patients (one patient is used to develop the $2 \mathrm{D}$ breast model). On the first dataset the proposed method achieved Jaccard= $81.1 \% \pm 6.1 \%$ and dice coefficient $=89.4 \% \pm 4.1 \%$ and on the second dataset Jaccard $=84.9 \% \pm 5.8 \%$ and dice coefficient $=92.3 \% \pm 3.6 \%$. These results are qualitatively comparable with the existing methods in the literature.

Keywords: Breast MRI, Breast Segmentation, Pectoral Segmentation, Computer Aided Diagnosis, Active Contours

\section{Introduction}

In 2014 there were 55,200 new cases of breast cancer diagnosed in the United Kingdom (UK). This approximates to 150 cases diagnosed every day on average, making breast cancer the most common cancer in the UK [1]. Similarly, in 5 Europe, this disease is also the most common cancer overall, with more than 460,000 new cases diagnosed in 2012; the highest and lowest incidence rates are in Belgium and Bosnia Herzegovina, respectively; and the UK breast cancer incidence rates are the $6^{\text {th }}$ highest in Europe [2]. Although the survival rates in most developed countries have been increasing in the last decade due to improved screening methods, much effort still needs to be invested in fighting this disease.

The use of computer-aided diagnosis (CAD) systems in medical applications as a 'second reader opinion' is becoming popular due to its consistency, reliability, and speed. However, before further analysis can be done, it is crucial for 15 the computer algorithm itself to capture regions of interest accurately. In breast $\mathrm{CAD}$, breast segmentation is an important first pre-processing step to speed up the subsequent processes without losing any important anatomical information 
[3, 4, 5, 6, 7, 8, 9, 10, 11]. For example, breast segmentation (estimating the breast boundary and chest wall boundaries) removes unnecessary regions such as the heart, lung and liver. This will narrow down the area to be analysed by the computer algorithm, hence speeding up the analysis process. On the other hand, in magnetic resonance imaging (MRI) volume assessment breast segmentation alone is important as an alternative measurement of breast density [5, 12, 13] and 3D breast reconstruction [14. For example, dense breasts tend to have higher volume/weight, and this is important information for the surgeon during the breast reconstruction. However, a fully automated method to estimate the breast and chest wall boundaries is a challenging task due to large variations in breast size and shape, intensity inhomogeneity, image artifacts and other noise errors [7.

30 The existing studies in the literature can be divided into three categories which are anatomical model-based, classifier-based, clustering-based and a hybrid method which is a combination of any of them. Each category has its own strength and weaknesses. For example a model-based method is efficient but relaying heavily on the model which can produce extremely poor results in cases where anatomical landmarks are invisible in the image. On the other hand, a classifier-based method can produce good results but there are many hyper-parameters need to be tuned and can be time consuming due to the training process. A clustering-based method is another alternative that can produce fast results but it is very sensitive to intensity inhomogeneity resulting

40 to over-segmentation or under-segmentation. A hybrid method can provide better results as it combines different advantages of each category such as efficient, less sensitive with noise and less dependent with anatomical landmarks. However it is often deemed to be semi-automatic (e.g., requires user initialisation or prior knowledge from the user) and requires more complex pre-processing (e.g., 45 image registration) and post-processing (e.g., curve smoothing).

To encounter some of the deficiencies in the existing studies, we present a fully automatic method for estimating the breast and pectoral muscle boundaries in MR images. The proposed method consists of the following phases: (a) 
pre-processing, (b) the development of a mathematical 2D breast model which covers landmark identification, (c) breast boundary estimation, (d) pectoral muscle boundary estimation and (e) post-processing. The novel contribution of our study is that we develop a simple mathematical 2D breast model and show how it can be used for breast segmentation in MR images in conjunction with active contour models (ACM). Furthermore, we studied how the inflation and deflation forces in active contour models can be used simultaneously to estimate the chest wall (pectoral muscle) boundary. To the best of our knowledge, this is the first time the Geodesic ACM model has been used in exploiting deflation and inflation forces applied to the breast segmentation problem. In comparison to the existing methods in the literature, our method is different in terms of:

1. Lower dependency on anatomical knowledge. Our method depends only on anatomical knowledge during the process of constructing the $2 \mathrm{D}$ breast model.

2. No prior knowledge is required during the segmentation phase.

3. No image registration step is required.

4. No clustering is needed. Our proposed method employs an ACM which evolves based on the appearance of gradient information close to the initial contour.

The paper is organised as follows: In Section 2 we review some of the existing methods in the literature followed by some variations of ACMs; Section 3 presents the technical aspects of the proposed method, covering the construction of the $2 \mathrm{D}$ breast model and the estimation of the breast and pectoral muscle boundaries. We present experimental results and discuss the effects of various parameter values on performance in Section 4. We discuss some of the limitations of the study, future directions, qualitative comparison with the existing methods in the literature and explanations about the weaknesses of the proposed method in Section 5. Finally, Section 6 summarises the work and presents some conclusions. 


\section{Literature Review}

This section will briefly describe some of the current studies in chest wall

\subsection{Related Work}

In comparison to breast segmentation in mammograms, the number of studies that have been undertaken to estimate both breast and pectoral muscle boundaries in MR images is relatively small. This is probably due to the lack of studies previously conducted by Giannini et al. 4, Wang et al. [3], GubernMérida [5, Rosado-Toro et al. 6], Thakran et al. 7], Milenković et al. 8], Fooladivanda et al. [9, Wu et al. [10] and Jiang et al. [11.

Early studies [19, 20, have attempted to segment the breast region using 95 a thresholding-based method followed by morphological operations which are efficient and could produce good results. However, in cases where breast intensity appears to be very similar to the other structures in the image due to noise or field inhomogeneities, a thresholding-based method may fail to segment the breast region. Giannini et al. 4 developed a method based on anatomical features of the pectoral muscle, which is detected given the assumption that the average muscle intensity is lower than that of fat within the breasts but higher than air in the lungs. Firstly, Otsu's thresholding method was used to separate the skin and breast parenchyma from internal and external air and other lowintensity areas. Secondly, a central point (initial point) of the breast boundary

105 is determined and, to estimate the pectoral muscle boundary, the method selects a pair of vertical points for each column that maximises the difference of gradient. Subsequently, this process is repeated until all corresponding points are 
covered. However, although this method works well in cases where the breast region is separated clearly from the pectoral region, if the region between the pectoral and breast boundary has a similar appearance or similar intensity to the pectoral region the method may fail to find the pectoral boundary due to insufficient or absent gradient information.

Wang et al. 3] used a Hessian-based filter call a 'sheetness' filter to analyse the local geometrical structure in the image. Their method assumes that the pectoral boundary has a similar appearance to sheet-like structures and this characteristic can be captured by tuning the parameters of Hessian-based filters. After employing 'sheetness' filters, only strong responses were retained using a thresholding value and false positives were removed using a connected component filter. This method may suffer from problems similar to the method of Giannini et al. 4, especially when the breast regions are dominated by fatty tissue rather than dense tissue. Another study in breast MRI segmentation was conducted by Rosado-Toro et al. [6] using $k$-means ++ and dynamic programming by exploiting the contrast properties in the fat and water images generated by a fat-water imaging method using the radial gradient- and spinecho (RADGRSE) technique. Dynamic programming is used to estimate the pectoral muscle boundary from the axial fat-image and the $k$-means ++ clustering algorithm was employed to segment the breast region. Finally, both results are combined to get the segmentation result.

The proposed method of Gubern-Mérida [5] was developed to automatically 130 capture breast density. In the breast segmentation phase, firstly image normalisation was performed to correct intensity inhomogeneities. Subsequently, a 3D probabilistic atlas-based approach was used to separate the breast from the air background. A morphological dilation filter is then applied to remove the skin between the background and the breast. In contrast, the pectoral muscle 135 boundary was estimated in a 3D model based on the location of the sternum. A coronal plane was defined at $2 \mathrm{~cm}$ posterior from the sternum landmark and excluded all the voxels which are posterior to this plane. Wu et al. [10] proposed a method based on edge-enhancing filters and an edge linking algorithm 
to estimate the chest wall in a sagittal view. timation using multi-parametric MR images. The authors used T2-Weighted (W), T1-W and Proton Density(PD)-W without fat saturation images with an automatic landmarks detection technique based on operations like profile screening, Otsu thresholding, morphological operations and empirical observation. ages based on the edge map (EM) obtained by applying an adaptive Gabor filter which initialise its parameters according to the local texture for detecting non-visible transitions between different tissues intensities. Subsequently, the shortest-path search technique in conjunction with a novel cost function is used to estimate the location of the pectoral muscle boundary. Fooladivanda et al. 9] used an intensity-based operation to estimate the location of the breast chest wall boundary. Based on shape and textural features, the support vector machine (SVM) is used to determine the connectivity of fibroglandular tissue to the chest wall. Finally, the initial boundary is refined using the geometric shape Finally, Jiang et al. [1] employed dynamic programming to trace the boundary of the chest wall starting from the middle slice and limit the search range using a Gaussian function.

\subsection{Active Contour Models}

ACMs are among the most popular segmentation techniques used in many different applications. In medical image analysis, the ACM has been widely used for the segmentation of brain and bone MR images, breast mammograms and retinal images. In this section, we briefly review several ACMs and their variations by dividing them into four categories, namely region-based active contours, edge-based active contours, level set models and hybrid models. We refer the reader to the study conducted by Lee [21] for more variations of ACM.

The study of Kass et al. [22] developed a model called "United Snakes" which aims to combine the best features of the various snake techniques, hence 
providing more flexibility and minimum user interaction. Ciecholewski 23 de-

veloped an $\mathrm{AC}$ model using an inflation/deflation force with a damping coefficient function to accurately estimate weak boundaries or edges in noisy regions. Álvarez et al. 24] developed an AC model based on morphological operators in conjunction with the Partial Differential Equation components, whereas Ferrari et al. [6] developed an adaptive active deformable contour model (AADCM) depending on the magnitude and direction of the image gradient. Wirth et al. [25] employed an AC model based on a Greedy algorithm which considers the energy from continuity, curvature and image gradient at each point. Miller et al. [26, 27. developed a model that firstly refined the estimated object's boundary by minimising the energy function followed by finding its actual boundary by taking account of the local contour curvature of the model and image features. This process was repeated discretely until the model reached an equilibrium state where velocity and acceleration are zero for each vertex.

Chan and Vese 28] developed an AC model based on techniques of curve evolution, the Mumford-Shah segmentation technique and level sets. The authors proposed a stopping function based on Mumford-Shah segmentation techniques instead of a gradient-based function, which enables the model to detect objects not defined by the gradient. Later, Li et al. 29] proposed a robust level set method that can deal with intensity inhomogeneities using a local clustering criterion function which is based on the local intensity clustering property. Zhang et al. 30, also proposed an AC model which attempts to deal with intensity inhomogeneities by introducing a local image fitting (LIF) energy function based on the local image information which minimises the difference between the fitted image and the original image. The LIF function is used to evolve the level set function and it is regularised with a novel Gaussian kernel filtering after each iteration in order to enhance the smoothing capacity.

Belaid et al. 31] used local phase and local orientation derived from the monogenic signal for the evolution of the level set approach to segment the left ventricle in ultrasound image. Furthermore, they used Cauchy kernels, instead of the commonly used log-Gabor, as pair of quadrature filters for the feature 

number of iterations $(i)$ is crucial to avoid under- or over-segmentation. 


\section{Methodology}

Figure 1 shows a graphical overview of the proposed method used in this study which consists of three main phases. After pre-processing using the me-

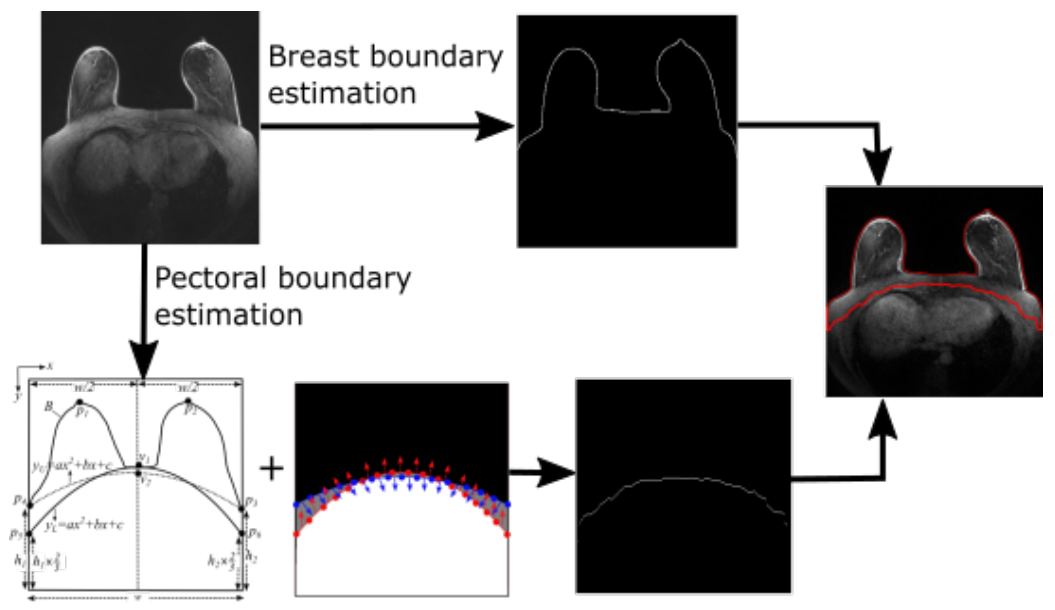

Figure 1: A graphical illustration of the proposed method.

dian filter and adaptive histogram equalisation [37, the first phase estimates the breast boundary based on the best edge features using a combination of Otsu's thresholding 38 and Canny's edge detection method. The second phase is to estimate the pectoral breast boundary using the $2 \mathrm{D}$ breast model in conjunction with the ACM of Caselles et al. 35. Finally, both estimated boundaries are smoothed and combined to separate the breast region from the pectoral muscle and the air background.

\subsection{A generalised 2D Breast Model}

To develop a generalised 2D breast model, it is important to identify important landmarks and a general shape (in the axial plane) of the breasts in MR images. For this purpose, we took $30 \mathrm{MRI}$ slices from a patient and performed an image fusion operation using $I_{o}=I_{1}+I_{2}+I_{3}+I_{4} \ldots . . I_{n}$ to generate a new fused image (in our case $n=30$ ). Due to the small number of data we selected only one patient (30 slices) so that we can maximise the number of patients 
manual annotation provided by one of the authors, yielding two images, namely a combined MR image $\left(I_{o}\right)$ and a combined manual annotation image $\left(I_{m}\right)$. Note that there is no selection criteria in choosing the patient when generating the $2 \mathrm{D}$ breast model. Any patient can be chosen because breast structures are similar across patients. The purpose of doing this is to get a general shape of a breast structure in a $2 \mathrm{D}$ axial MR image. However, for slice selection it is recommended to take the middle slices as these slices contain the most visible shape of the breast and chest wall boundaries. The slice selection refers to the process of choosing a set of images from the middle slices in order to construct a summed image $\left(I_{O}\right)$.

Figure 2 shows a general overview used to construct the 2D model for breast appearance in MR images. The images $I_{o}$ and $I_{m}$ (see figure 3.2 enable us to identify important features of the breast and we use empirical observation knowledge to model the general appearance of the breasts in MR images.

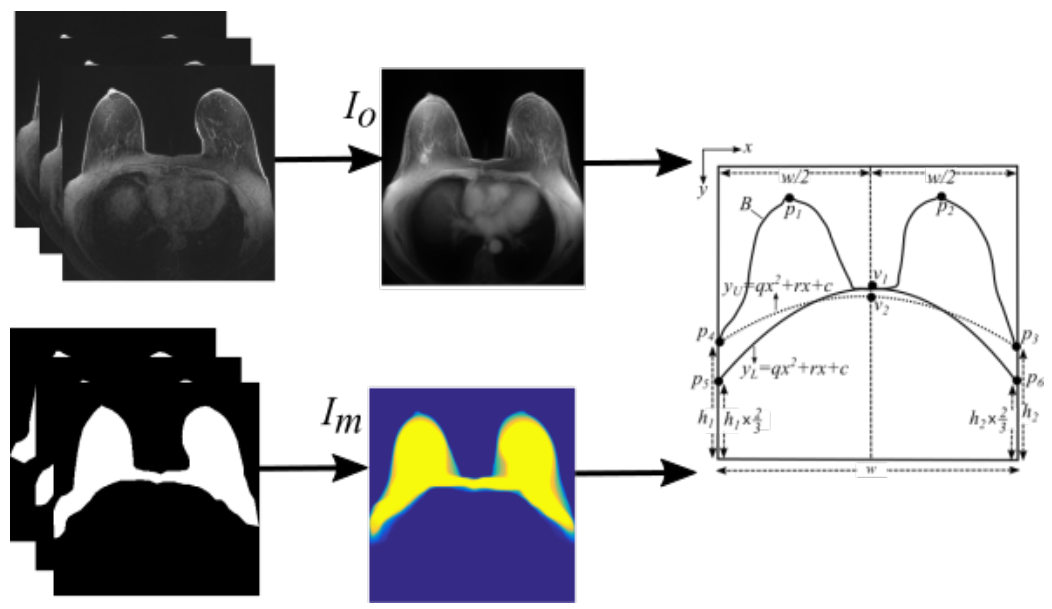

Figure 2: A work-flow for modelling the breast appearance in MR images

Figure 3.2 shows image $I_{m}$ (left) and our 2D breast model (right). Let $B$ be the estimated breast boundary (see Figure 3.2 represented as a set of coordinate points, hence $B=\left\{\left(x_{1}, y_{1}\right),\left(x_{2}, y_{2}\right) \ldots\left(x_{\max }, y_{\max }\right)\right\}$ where $x$ and $y$ 
are the row and column coordinate respectively. Now let $B_{x}$ and $B_{y}$ contain all the $x$-coordinate and $y$-coordinate points in $B$. Based on $I_{m}$, we identified the following characteristics:
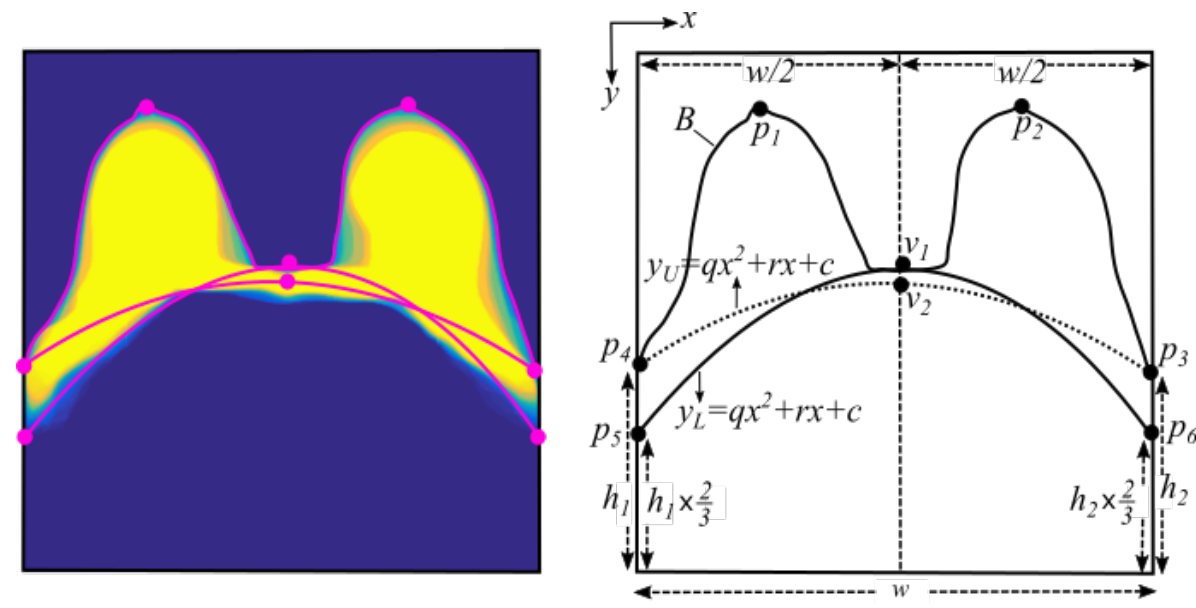

Figure 3: A graphical illustration for the 2D model of the breast appearance

1. Each breast is located in half of the width $(w)$ of the image.

2. The breast boundary contains two peaks. The first peak $\left(p_{1}\right)$ can be captured by taking the highest point in the first half of the image (left side of the MR image) and the second peak $\left(p_{2}\right)$ is the highest point in the second half of the image (right side of the MR image) as defined in equations 1 and 2 .

$$
\begin{gathered}
p_{1}=\left(B_{x}\left(l_{B_{y}^{\text {min }}}^{\text {left }}\right), \min \left(B_{y}\left(1: \# B_{y} / 2\right)\right)\right) \\
p_{2}=\left(B_{x}\left(l_{B_{y}^{\text {min }}}^{\text {right }}\right), \min \left(B_{y}\left(\# B_{y} / 2+1: \text { end }\right)\right)\right)
\end{gathered}
$$

where $l_{B_{y}^{\text {min }}}^{l e f t}$ and $l_{B_{y}^{\text {min }}}^{\text {right }}$ are the location of the minimum value of $B_{y}$ in the first and second halves of the image, respectively, where $\# B_{y}$ is the number of elements of $B_{y}$.

3. The breast boundary has a global valley point $\left(v_{1}\right)$ which is the lowest 
point on $B$ between $p_{1}$ and $p_{2}$ defined using the following equation

$$
v_{1}=\left(B_{x}\left(l_{B_{y}^{\text {max }}}^{\text {middle }}\left(p_{1}: p_{2}\right)\right), \max \left(B_{y}\left(p_{1}: p_{2}\right)\right)\right)
$$

where $l_{B_{y}^{\text {max }}}^{\text {middle }}$ is the location of the maximum value of $B_{y}$ from $p_{1}$ to $p_{2}$;

4. The pectoral boundary can be approximated using the following quadratic equation

$$
y_{U}=q x^{2}+r x+c
$$

This is the dotted curve in the right image of Figure 3.2 . This equation can be solved by substituting coordinates of $p_{4}, v_{2}$ and $p_{3}$ into the quadratic equation. All points can be calculated using the following equations

$$
\begin{gathered}
p_{3}=\left(B_{x}(\text { end }), B_{y}(\text { end })\right) \\
p_{4}=\left(1, B_{x}(1)\right) \\
v_{2}=\left(v_{1}^{x}, v_{1}^{y}+d\right)
\end{gathered}
$$

where $v_{1}^{x}$ is the $x$-coordinate of $v_{1}$ and $v_{1}^{y}+d$ is the $y$-coordinate of $v_{1}$ with respect to parameter $d$, which is the vertical distance between $v_{1}$ and $v_{2}$. Note that from equations 5 and 6 both $p_{3}$ and $p_{4}$ are the last and first coordinate points in $B$, respectively. Alternatively, $p_{3}$ is a point in $B$ with the maximum $\mathrm{x}$-coordinate located in the second half of the image (right side), whereas $p_{4}$ is a point in $B$ with minimum $\mathrm{x}$-coordinate located in the first half of the image (left side).

$$
y_{L}=q x^{2}+r x+c
$$


This equation can be solved by substituting coordinates of $p_{5}, v_{1}$, and $p_{6}$ into the quadratic equation. All points can be calculated using the following equations

$$
\begin{gathered}
h_{1}=p_{4}^{y}-y_{\max } \\
h_{2}=p_{3}^{y}-y_{\max } \\
p_{5}=\left(1, p_{4}^{y}+\left(h_{1} / 3\right)\right) \\
p_{6}=\left(1, p_{3}(y)+\left(h_{2} / 3\right)\right)
\end{gathered}
$$

where $y_{\max }$ is the height of the image. Note that the $y$-coordinates for $p_{5}$ and $p_{6}$ are estimated to be about $30 \%$ below the $y$-coordinates of $p_{4}$ and $p_{3}$, respectively. This is based on our observation in $I_{o}$ and $I_{m}$ where the majority of the left and right sides of the pectoral muscles are located above this boundary $\left(y_{L}\right)$.

At the end of this process, our 2D breast model can be seen in the right image of Figure 3.2 This model presents the general shape appearance of the breast in MR images. In Sections 3.2 and 3.3 we will show how the model can be used as a 'template' to identify important landmark points along the breast boundary, yielding a solution for curves $y_{U}$ and $y_{L}$.

\subsection{Breast Boundary Estimation}

In this section, we will explain how the breast boundary which is the $B$ curve in the $2 \mathrm{D}$ model can be determined using a simple edge detection technique. Subsequently, we will show how the model can be used to identify important landmark points along the $B$ curve. For breast boundary estimation we employed Otsu's thresholding [38] and Canny edge detection to get all possible edge candidates (middle image in Figure 4). Subsequently, we retain the top 
three longest edges (fourth image in Figure 4) from the binary image and delete all other edges. Note that selecting the top five or more is possible, but in our case we found that all edges representing the breast boundaries can be captured by taking the top three longest edges. Finally, we select the edge which has the 315 smallest y-coordinate point as it indicates the location of the breast boundary that separates the breast from the air background. Figure 4 shows the step by step process in finding the breast boundary. Note that the edge detected along the breast boundary in the right-most image represents the $B$ curve in the 2D model. Using the 2D model in Figure as a 'template' we can identify other landmarks along the breast boundary such as the $P_{4}, P_{1}, v_{1}, P_{2}$ and $P_{3}$. Subsequently, the coordinate information of these points will be used to solve curves $y_{U}$ and $y_{L}$.

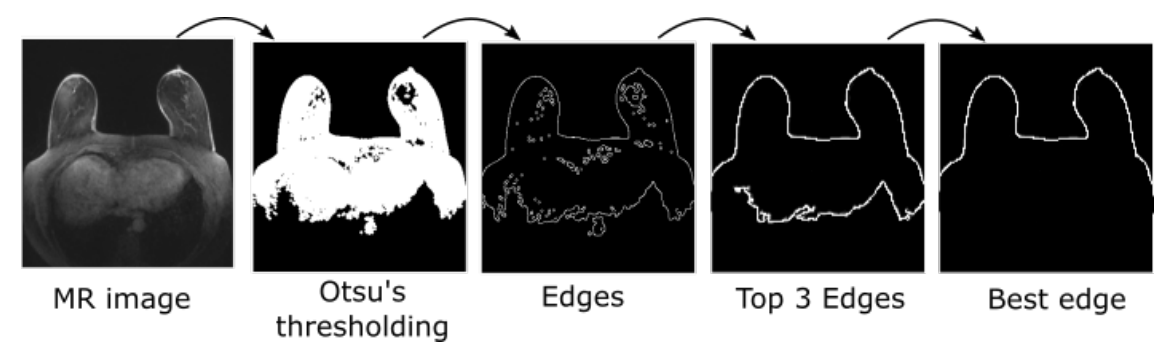

Figure 4: A graphical illustration for the breast boundary estimation.

\subsection{Pectoral Muscle Boundary Estimation}

In our case, the initial contours are the two quadratic equations (defined $y_{U}$ and $y_{L}$ in the previous section) are denoted as $y_{U}^{0}$ and $y_{L}^{0}$ where the desired boundary is the pectoral muscle boundary. In this section we use the notation curve as $\mathcal{C}(q)$ to cover $y_{U}^{0}$ and $y_{L}^{0}$ for simplicity. Note that $q$ is the arch length parameterisation. $\mathcal{C}_{0}$ and $\mathcal{C}_{i}$ represents the initial contour and the contour at $i^{\text {th }}$ iteration. Let $\mathcal{C}(q):[0,1] \rightarrow \mathbb{R}^{2}$ be parameterised planar curve. The classical edge-based snake approach [39] minimises the energy of curve $\mathcal{C}$ at $i^{\text {th }}$ iteration 
by computing the following equation:

$$
E\left(\mathcal{C}_{i}\right)=p \int_{0}^{1}\left|\left(\mathcal{C}^{\prime}{ }_{i}(q)\right)\right|^{2} d q+\lambda \int_{0}^{1}\left|\left(\mathcal{C}^{\prime \prime}{ }_{i}(q)\right)\right|^{2} d q-b \int_{0}^{1}\left|\nabla I\left(\mathcal{C}_{i}(q)\right)\right| d q
$$

where $p, \lambda$ and $b$ are the smooth factor, weight and contraction bias, respectively. The first two terms (note that $C_{i}^{\prime}$ and $C_{i}^{\prime \prime}$ are first and second derivative, respectively ) control the smoothness of the contours to be detected (internal energy), while the third term is responsible for attracting the contour towards the object in the image (external energy) 39, 35. In contrast, the GACM minimises the energy of curve $\mathcal{C}$ at $i^{\text {th }}$ iteration using the following equation:

$$
E\left(\mathcal{C}_{i}\right)=p \int_{0}^{1}\left|\mathcal{C}^{\prime}{ }_{i}(q)\right|^{2} d q+b \int_{0}^{1} g\left(\left|\nabla I\left(\mathcal{C}_{i}(q)\right)\right|\right)^{2} d q
$$

where $g\left(\left|\nabla I\left(\mathcal{C}_{i}(q)\right)\right|\right)^{2}$ is a function that controls the $\mathcal{C}$ attraction toward lines and edges. Note that $g:[0, \infty) \rightarrow \mathbb{R}^{+}$is a decreasing function such that $g(r) \rightarrow 0$.

Therefore

$$
g(r)=\frac{1}{1+\left|\nabla I\left(\mathcal{C}_{i}(q)\right)\right|^{2}}
$$

We employed the GACM of Caselles et al. [35, which is a variant of edgebased ACMs, in conjunction with our 2D breast model to estimate the pectoral muscle boundary. Our motivations in using GACM are three-fold:

1. GACM does not depend on curve parametrisation, which means the curve deformation is not restricted to curve parameters. Other edge-based ACMs such as 'snakes models' are usually parametrised using B-splines which constrain the curve deformation to a certain shape 35]. In our case, curve parametrisation is not needed anyway because the initial curves (e.g. $y_{U}^{0}$ and $y_{L}^{0}$ ) are determined automatically based on the location of $p_{3}, p_{4}$, $p_{5}, p_{6}, v_{1}$ and $v_{2}$.

2. GACM is robust in handling topology changes in the image whereas most of the edge-based ACMs require topology-handling procedures [35, 40, 
3. Region-based ACMs usually require segmentation techniques which can be very difficult to cluster due to similar intensity between the lower region of the breast and the pectoral muscle. In fact, the clustering process is time consuming because every corresponding point within the breast region is taken into account. In comparison, GACM computes only the neighbourhood points along the contour [40].

One of the key factors in using edge-based ACMs successfully is the placement of the initial contour, which must be close to the actual boundary. For this purpose, we used the knowledge from our 2D model to estimate the initial contour. Based on the breast contour defined in Figure 4 (leftmost image), we calculate important landmarks/vertexes such as $p_{1}, p_{2}, p_{3}, p_{4}, p_{5}, v_{1}, v_{2}$, etc. Once all coordinate points for these vertexes were identified, we can define $y_{U}^{0}$ and $y_{L}^{0}$ by substituting each vertex's $x$ - and $y$-coordinate into the corresponding equation (e.g. $y_{U}$ and $y_{L}$ ).

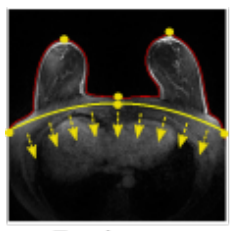

Evolve $y_{U}$

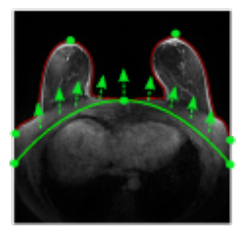

Evolve $y_{L}$

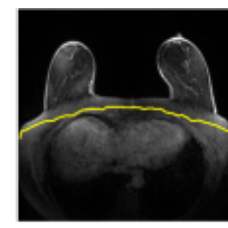

After evolution

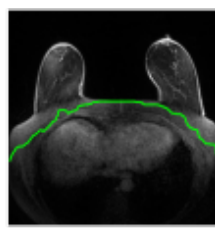

After evolution

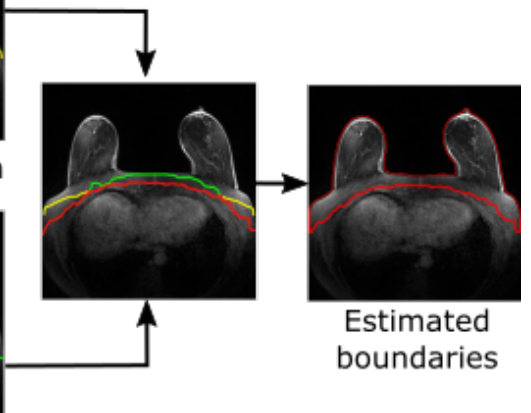

Figure 5: A work flow graphical representation for pectoral muscle estimation. The arrow directions in the leftmost images show the tendency of the initial contour to grow.

Figure 5 shows the steps involved in estimating the pectoral muscle boundary. Using the GACM [35], all coordinate points in $y_{U}^{0}$ (yellow line in Figure 5 ) and $y_{L}^{0}$ (green line in 5) are evolved separately using GACM. This means 370 the GACM was run twice independently, firstly using $y_{U}^{0}$ with deflation a force 
followed by $y_{L}^{0}$ with an inflation force. Both forces can be obtained by setting different values of the contraction bias parameter $(b)$. This parameter controls the tendency of the contour to grow outwards (inflation) or shrink inwards (deflation) with a typical value between -1 to 1 , where a positive value means the contour tends to grow outward (inflate). In our case, using these forces ensure the model is able to capture the upper boundary of the pectoral muscle and boundary around the chest wall. Therefore, in our case, we set $b=1$ and $b=-1$ when evolving $y_{L}^{0}$ and $y_{U}^{0}$ using the GCAM, respectively. Subsequently, after contour evolution, both $y_{L}^{0}$ and $y_{U}^{0}$ were combined and the lowest points in $y_{L}^{0}$ and $y_{U}^{0}$ representing the estimated pectoral boundary (see the rightmost image in Figure 5). Finally, we smooth the estimated pectoral boundary using a moving median filter to remove outliers.

\subsection{Materials and Datasets}

To test the performance of the proposed method, our first dataset was downloaded from the National Biomedical Imaging Archive (NBIA) 41] under the Quantitative Imaging Network Collection (QIN) of breast Dynamic Contrast Enhanced (DCE) MRI [42]. The DCE-MRI data was collected from Oregon Health \& Science University and covers 10 patients which were acquired using a Siemens 3 Tesla system with Syngo MR B17 software. DCE-MRI acquisition parameters included $10^{\circ}$ flip angle, 2.9/6.2 ms TE/TR, a parallel imaging acceleration factor of two, $30-34 \mathrm{~cm}$ FOV, slice thickness is $1.39 \mathrm{~mm}$ and $320 \times 320$ in-plane matrix size. The $x$ and $y$ resolution is $1.06 \mathrm{~mm}$ and $z$ resolution is $1.4 \mathrm{~mm}$. However, since $30 \mathrm{MRI}$ slices of a patient were used to build the $2 \mathrm{D}$ breast model, these images were excluded in evaluating the proposed method.

395 Therefore, the test database consists of 9 patients. The MR scans included in the QIN database are all fat-suppressed images which means fatty areas are contrast-enhanced making it easier for radiologists to visually identify fatty regions on each image slice. Both pectoral and breast boundary annotations on each image are provided by one of the authors and verified by an expert radiologist. 
The second dataset (our own data) consists of 50 patients of T2-W MRI collected from our clinical partner Hospital General Universitari Valencia and acquired using a Siemens 1.5 Tesla system with Syngo MR A35 software. The T2-W MRI acquisition parameters included $150^{\circ}$ flip angle, echo time: $114 \mathrm{~ms}$, repetition time: $3.350-5.950 \mathrm{~ms}$, slice thickness is $4 \mathrm{~mm}$ and $1024 \times 1024$ inplane matrix size. The $x$ and $y$ resolution is $0.35 \mathrm{~mm}$ and $z$ resolution is $4.4 \mathrm{~mm}$. Both pectoral and breast boundary annotations on each image are provided by an expert radiologist with more than 10 years experience in reading breast MRI. The method was developed under the MATLAB environment version 9 (2016a) on a Windows 10 operating system with an intel CORE i7 vPro processor.

\subsection{Evaluation Metrics}

To evaluate the performance of the proposed method we used the following metrics (more details of Jaccard and Dice metrics can be found in [43] and [44], respectively):

$$
\begin{gathered}
\operatorname{Jaccard}(\ddot{J})=\frac{\left|S_{I} \cap R_{I}\right|}{\left|S_{I} \cup R_{I}\right|} \\
\operatorname{Dice}(\ddot{D})=\frac{2\left|S_{I} \cap R_{I}\right|}{\left|S_{I}\right|+\left|R_{I}\right|} \\
\operatorname{Accuracy}(\ddot{A})=\frac{T P+T N}{T P+T N+F N+F P} \\
\text { Sensitivity }(\ddot{S})=\frac{T P}{T P+F N} \\
\text { Specificity }(\bar{S})=\frac{T N}{T N+F P}
\end{gathered}
$$

where TP, TN, FP and FN are true positive, true negative, false positive and false negative, respectively. $S_{I}$ and $R_{I}$ are the segmented image produced by the proposed method and the reference image or manual segmentation, respectively. The Hausdorff distance $(\nabla D)[45$ is used to measure the 'closeness' of two sets of points that are subsets of a metric space. Given two coordinate sets $A$ and $B$ 
in Euclidean space, the Hausdorff distance can be calculated using the following equation

$$
\begin{gathered}
\nabla D=\operatorname{dist}_{B}(A)+\operatorname{dist}_{A}(B) \\
\operatorname{dist}_{B}(A)=\sup _{a \in A} \operatorname{dist}(a, B) \\
\operatorname{dist}(a, B)=\inf _{b \in B} d(a, b)
\end{gathered}
$$

where $a$ and $b$ are each points in set $A$ (the boundary of the segmented region) and $B$ (the boundary of the manual annotation), respectively. For all the evaluation metrics used in this study, a higher value indicates better performance;

${ }_{425}$ however, for $\nabla D$ a smaller value means the estimated boundary is closer to the manual annotation.

\section{Experimental Results}

This section will first briefly describe the experimental results in Subsections 4.1. Next, we discuss the effects of two parameters in GACM, namely the contraction bias $(b)$ and smoothing $(s)$. We perform a statistical analysis using the $t$-test by comparing each result with the average result across different thresholds. For example, when comparing accuracy the average result can be computed by summing all accuracies across different threshold values followed by dividing it with the number of thresholds. The $t$-test approach is 435 used to compare each accuracy produced at a specific threshold value against the average accuracy.

\subsection{Quantitative Results}

Since the proposed method employed GACM [35, there are several parameters which must be defined, namely vertical distance between $v_{1}$ and $v_{2}(d)$,

${ }_{440}$ the number of iterations for the active contours to evolve $(i)$, contraction bias 
(b) which controls the tendency of the contour to evolve inwards or outwards, and a smoothing parameter $(s)$ which controls the smoothness of the contour. The values chosen for these parameters are important in order to get optimal results. Therefore, this section presents the results from all metrics when choosing different values for each parameter. For this purpose, we chose the QIN dataset due to its smaller number of images, hence speeding up the process of parameter selection.

To investigate the effect of $i$ on the proposed method, we conducted an experiment using 30 different values from $i=5$ to $i=150$ at intervals of five. Other parameters were set as $d=10, s=1.0$ and $b=-1$ for $y_{U}^{0}$ and $b=1$ for $y_{L}^{0}$. These parameter values were chosen because they produce optimal performance of the proposed method as shown in Figures 6, 7, 10 and 11 . Note that each point on the graphs is an average across the 270 images. Figure 6 show the performance variations for all metrics when different numbers of iterations $(i)$ are used for the QIN dataset. It can be observed that both $\ddot{J}$ and $\ddot{D}$ start to decrease when $i>100$, which indicates that the pectoral boundary was overestimated and maximum results achieved at $i=100$ for both metrics. The proposed method produced consistent results for values of $i$ between 70 and 100 with $\ddot{J}>80 \%$ and $\ddot{D}>88 \%$. Similar results can be observed in Figure 6 for $\ddot{A}$ and $\ddot{S}$ where consistent results can be obtained using values of $i$ between 70 and 100. However, all metrics started to decrease after $i>75$ with the best results achieved for $\ddot{A}$ and $\ddot{S}$ at $i=75$. For metric $\nabla D$, Figure 7 shows that the smallest distance $(\nabla D=5.71 \mathrm{~mm} \pm 2.64 \mathrm{~mm})$ was obtained using $i=75$ and increasing the value of $i$ results in a larger difference between the segmentation result and the manual segmentation. Overall, the proposed method produced consistent results across different metrics for values of $i$ between 70 and $100(\ddot{J}>80 \%$, $\ddot{D}>86 \%, \ddot{A}>95 \%, \ddot{S}>85 \%$ and $\bar{S}>97 \%$. In terms of the evaluation of the proposed generalised 2D mathematical model the average distances are $\nabla D=3.84 \mathrm{~mm} \pm 2.29 \mathrm{~mm}$ and $\nabla D=3.98 \mathrm{~mm} \pm 2.38 \mathrm{~mm}$ for the QIN and our 470 own datasets, respectively. The evaluation was measured using the Hausdorff Distance for each of the estimated breast boundary with its associated ground 
truth.
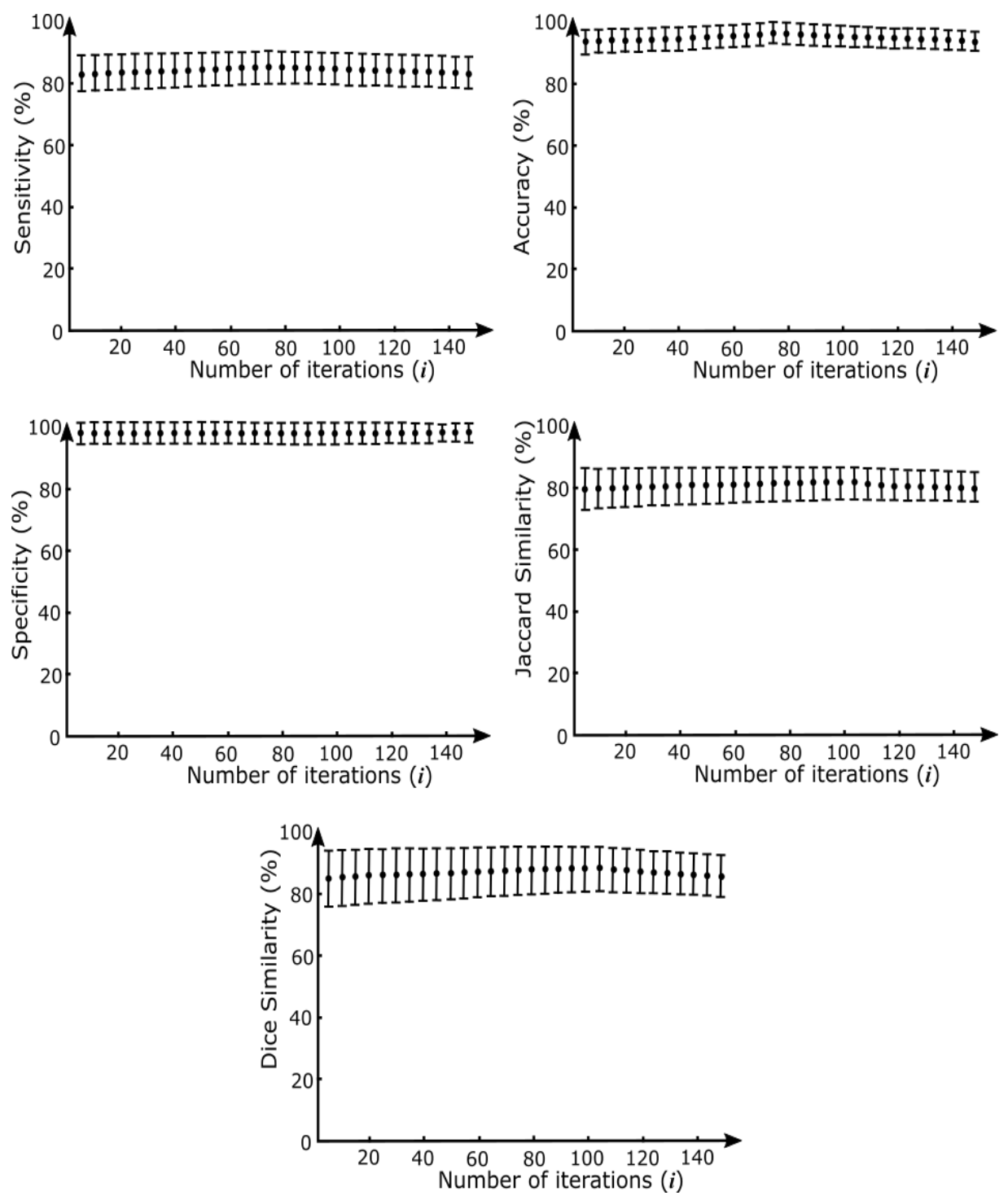

Figure 6: Performance variation of metrics $\ddot{J}$ and $\ddot{D}, \ddot{A}, \ddot{S}$ and $\bar{S}$ with standard deviation using a different number of iterations $(i)$ for the QIN dataset

We also tested the effect of the vertical distance between $v_{1}$ and $v_{2}$ by using the following set of values for $d:\{5,10,15,20\}$. In this experiment, we set 


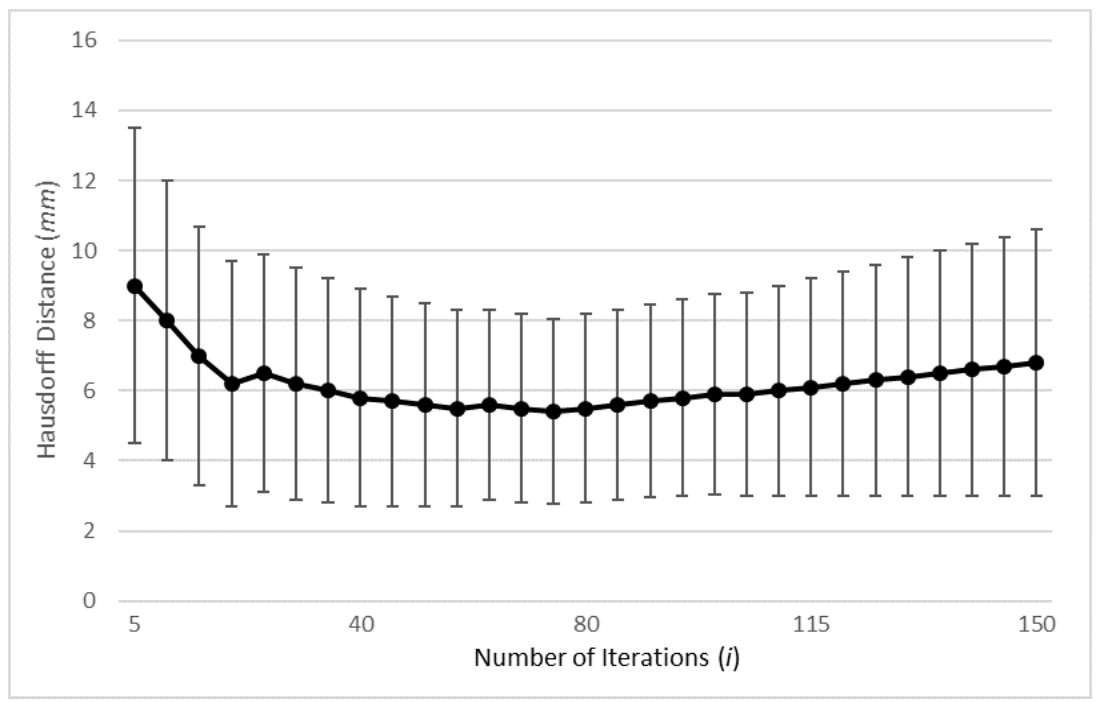

Figure 7: Performance variation of metric $\nabla D$ with standard deviation using a different number of iterations $(i)$ for the QIN dataset.

the other parameters as follow: $i=100, s=1.0, b=-1$ for $y_{U}^{0}$ and $b=1$ for $y_{L}^{0}$. Table 1 shows quantitative results for the proposed method when using different values of $d$. In general, the method produced better results using $d=5$ and $d=10$ than for $d=15$ and $d=20$. This is because locating $v_{2}$ too far away from $v_{1}$ results in $y_{U}^{0}$ being closer to the chest wall instead of the pectoral muscle (hence, it tends to move closer to the chest wall instead). From the four different values of $d$ tested, the results in Table 1 suggest that $d=5$ or $d=10$ produced the best results across the different metrics.

Figure 8 shows examples of segmentation results with good values across different evaluation metrics. The left-hand, middle and right-hand columns are the original, ground truth (manual segmentation in binary image) and the segmentation results of the proposed method, respectively. Evaluation metrics are presented to the right of each row. The red and green lines indicate the breast and pectoral muscle estimation, respectively. Both quantitative and qualitative evaluation in Figure 8 shows that the proposed model is robust in conjunction with GACM to estimate both breast and pectoral muscle boundaries. Examples 
Table 1: Quantitative results using different values of $d$ (the vertical distance between $v_{1}$ and $v_{2}$ in Figure 3.2 for QIN dataset.

\begin{tabular}{ccccccc}
\hline$d$ & $\ddot{J}(\%)$ & $\ddot{D}(\%)$ & $\ddot{A}(\%)$ & $\ddot{S}(\%)$ & $\bar{S}(\%)$ & $\nabla D(\mathrm{~mm})$ \\
\hline \hline 5 & $80.8 \pm 6.7$ & $89.3 \pm 4.4$ & $96.3 \pm 1.4$ & $88.1 \pm 4.9$ & $97.8 \pm 2.1$ & $6.2 \pm 2.6$ \\
10 & $80.9 \pm 5.9$ & $89.4 \pm 3.8$ & $96.2 \pm 1.2$ & $86.2 \pm 6.2$ & $98.4 \pm 1.8$ & $6.2 \pm 2.6$ \\
15 & $79.4 \pm 6.6$ & $88.3 \pm 4.4$ & $95.3 \pm 1.4$ & $83.2 \pm 8.2$ & $97.9 \pm 2.4$ & $6.6 \pm 2.7$ \\
20 & $76.6 \pm 7.9$ & $86.5 \pm 5.5$ & $94.2 \pm 1.9$ & $79.5 \pm 9.3$ & $98.1 \pm 1.4$ & $7.2 \pm 2.9$ \\
\hline
\end{tabular}

in Figure 8 show different variations in terms of shapes and textures of the breast. In the second and fifth row, the pectoral muscle is almost invisible due to similar intensities between the breast region and the pectoral muscle, where the proposed method managed to achieve $\ddot{J}=89.6 \%$ and $86.3 \%$, respectively. In the fourth row, although the orientation of the breast is slightly to the right, the proposed method achieved $\ddot{J}=83.2 \%$ and $\ddot{D}=90.8 \%$. Figure 9 presents examples of estimated breast (red line) and chest wall (green) boundaries taken from the second dataset (our own dataset). All examples show good results in estimating both boundaries.

\subsection{The Effect of Contraction Bias}

In the proposed method, two contours $\left(y_{L}^{0}\right.$ and $\left.y_{U}^{0}\right)$ were employed each using different forces (inflation and deflation) which were controlled by the parameter $b$. To investigate the effect of $b$, we conducted an experiment by varying its values from $b=0.1$ to 1.0 (inflation) and $b=-0.1$ to -1.0 (deflation) for $y_{L}^{0}$ and $y_{U}^{0}$, respectively, at intervals of 0.1 . The effects on the performance as presented in Figure 10 were measured for $\ddot{J}, \ddot{D}, \ddot{A}, \ddot{S}$ and $\bar{S}$. Nevertheless, it can be observed that the performance of the proposed method improved slightly $(\approx 1 \%)$ for metrics $\ddot{J}, \ddot{D}$ and $\ddot{S}$ from $b=0.1(-0.1)$ to $b=0.6(-0.6)$, with no further changes when $b>0.6(<-0.6)$. In our study we chose $b=1.0(-1.0)$ as default values for ease of numerical calculation. Experimental results suggest that there is no statistical significant of improvement for all evaluation metrics across the 

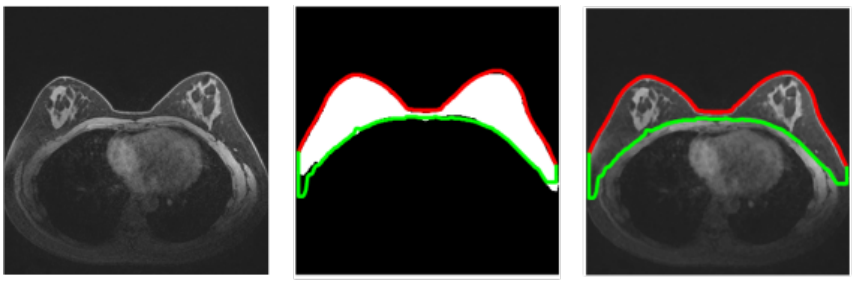

Jaccard $=86.4$

Dice $=92.7$

Sensitivity $=88.2$

Accuracy $=97.9$

Specificity $=99.6$

Hausdorff Distance $=6.8$
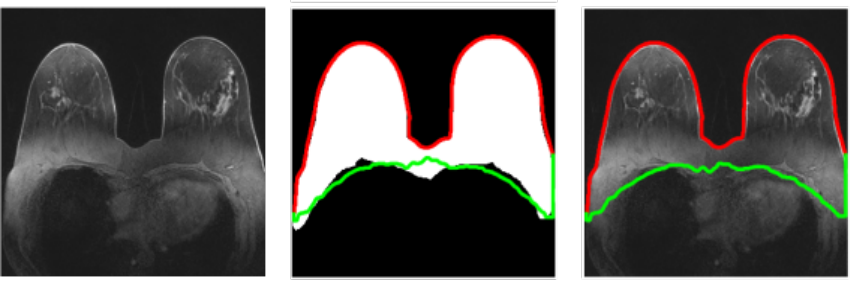

Jaccard $=89.6$

Dice $=94.5$

Sensitivity $=92.6$

Accuracy $=96.1$

Specificity $=98.1$

Hausdorff Distance $=5.9$
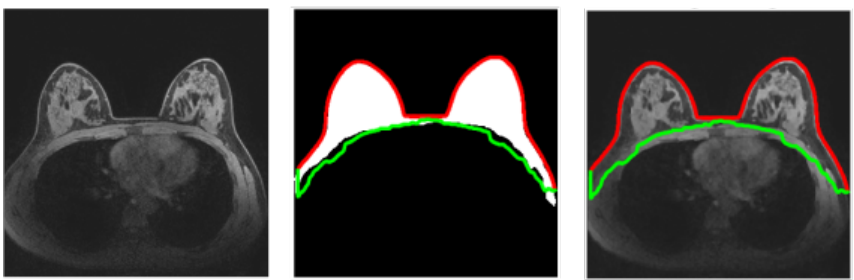

Jaccard $=82.6$

Dice $=90.5$

Sensitivity $=84.4$

Accuracy $=97.1$

Specificity $=99.5$

Hausdorff Distance $=4.7$
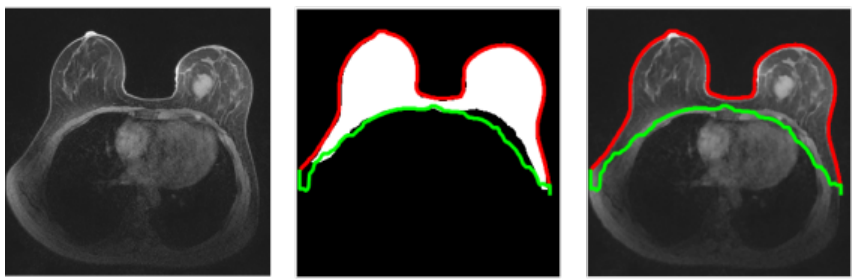

Jaccard $=83.2$

Dice $=90.8$

Sensitivity $=84.1$

Accuracy $=96.5$

Specificity $=99.6$

Hausdorff Distance $=9.1$
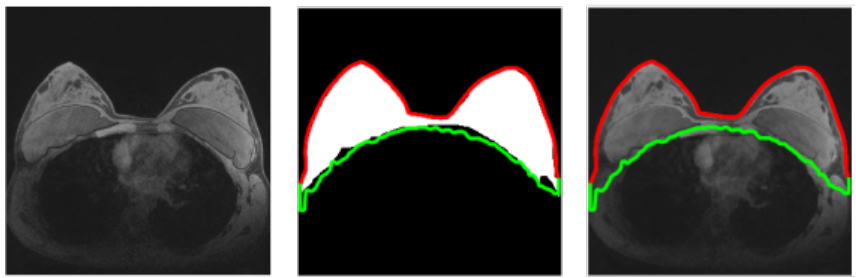

Jaccard $=86.3$

Dice $=92.6$

Sensitivity $=86.3$

Accuracy $=97.1$

Specificity $=99.5$

Hausdorff Distance $=5.3$
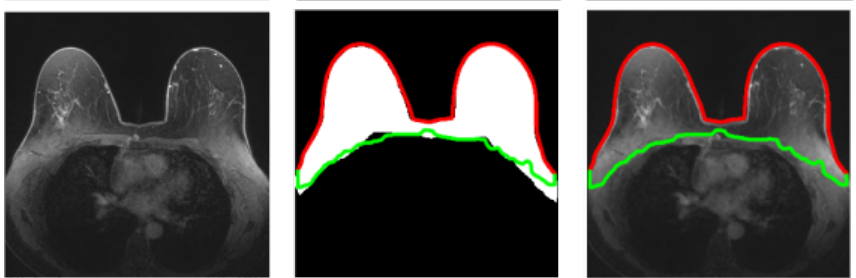

Jaccard $=88.3$

Dice $=93.8$

Sensitivity $=92.7$

Accuracy $=97.1$

Specificity $=98.5$

Hausdorff Distance $=5.5$

Figure 8: Example segmentation results for breast (red) and pectoral muscle (green) boundary estimation with high $\ddot{J}$ and $\ddot{D}$ more than $80 \%$ taken from the QIN dataset. 

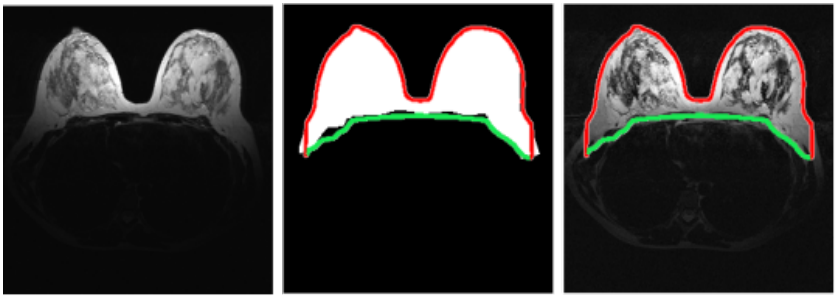

Jaccard $=88.4$

Dice $=94.2$

Sensitivity $=89.9$

Accuracy $=97.3$

Specificity $=99.6$

Hausdorff Distance $=5.1$
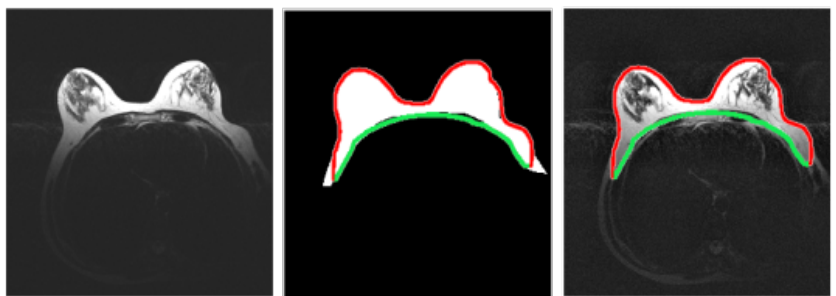

Jaccard $=85.4$

Dice $=93.9$

Sensitivity $=89.2$

Accuracy $=97.1$

Specificity $=99.5$

Hausdorff Distance $=5.4$
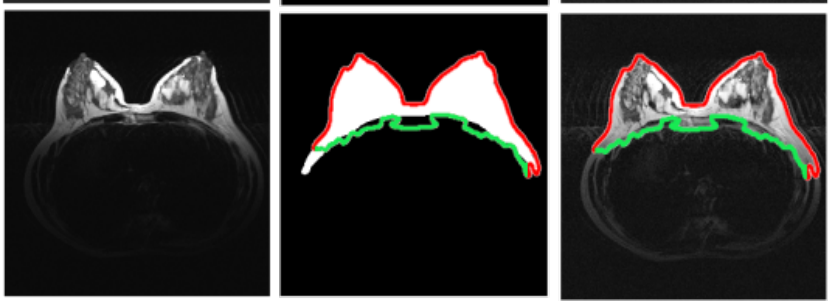

Jaccard $=80.4$

Dice $=89.9$

Sensitivity $=84.1$

Accuracy $=93.2$

Specificity $=97.2$

Hausdorff Distance $=7.1$

Figure 9: Example segmentation results for breast (red) and pectoral muscle (green) boundary estimation with high $\ddot{J}$ and $\ddot{D}$ more than $80 \%$ taken from our dataset.

values of $b$ (at $p<0.01$ ) with $p=0.0507$. The $p$ value was computed using $t$-test comparing each result with the average result across different parameter values. This suggests that the results are very consistent regardless of the $(b)$ value (variation results are from $0.1 \%$ to $0.7 \%$ ).

\subsection{The Effect of Smoothing Parameter}

In addition, we were also interested to know the effect of parameter $s$ in our study which controls the degree of smoothness or regularity of the contour of the segmented regions (in our case it is the pectoral boundary). Using a large value of $s$ produces smooth contours but may miss finer details, whereas a small value will produce irregular contours but allow finer details to be captured. We conducted an experiment by varying the values of $s$ from 0.1 to 1.0 at increments of 0.1 . Figure 11 shows the results across different metrics which indicate that 

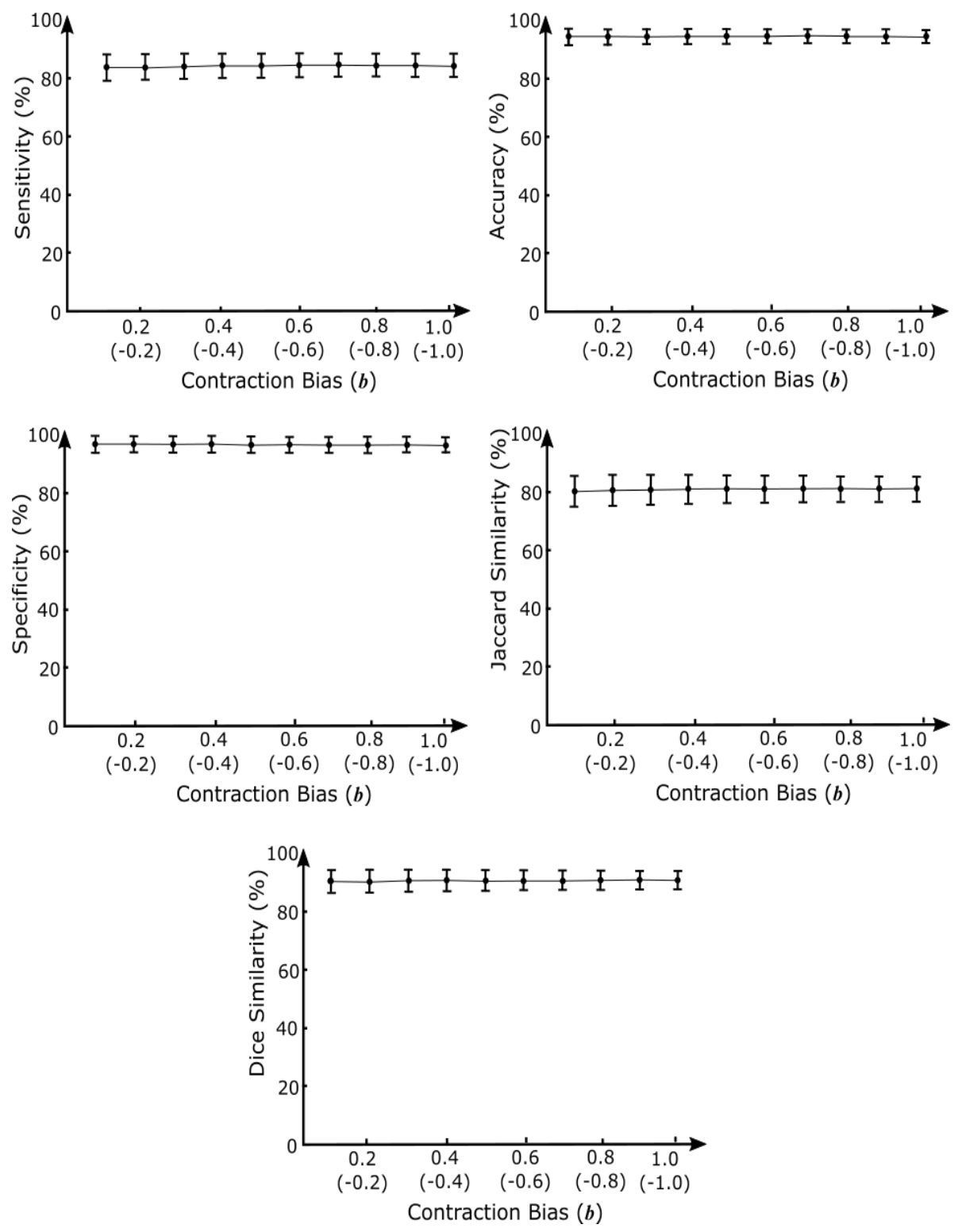

Figure 10: Performance variation of metrics $\ddot{J}, \ddot{D}$ and $\ddot{A}, \ddot{S}$ and $\bar{S}$ with standard deviation using different values of contraction bias $(b)$ for the QIN dataset. Note that the $\mathrm{x}$ - axis has positive and negative values for $y_{L}^{0}$ and $y_{U}^{0}$, respectively. Note that small variations on performance indicate consistency across different values of $b$. 
the smoothing parameter does not have a significant effect on the performance.

${ }_{525}$ This may be due to (a) the use of the median filter to smooth the pectoral boundary in the post-processing phase and (b) most pectoral boundaries having a smooth appearance resulting in a smooth contour. For the ease of numerical calculation, we used a default value $s=1.0$. Once again, we found $p=0.0507$ which indicates no statistical significant improvement for all evaluation metrics ${ }_{530}$ across different values of $s$ at $p<0.01$ (variation results are from $0.1 \%$ to $0.6 \%$ ). 

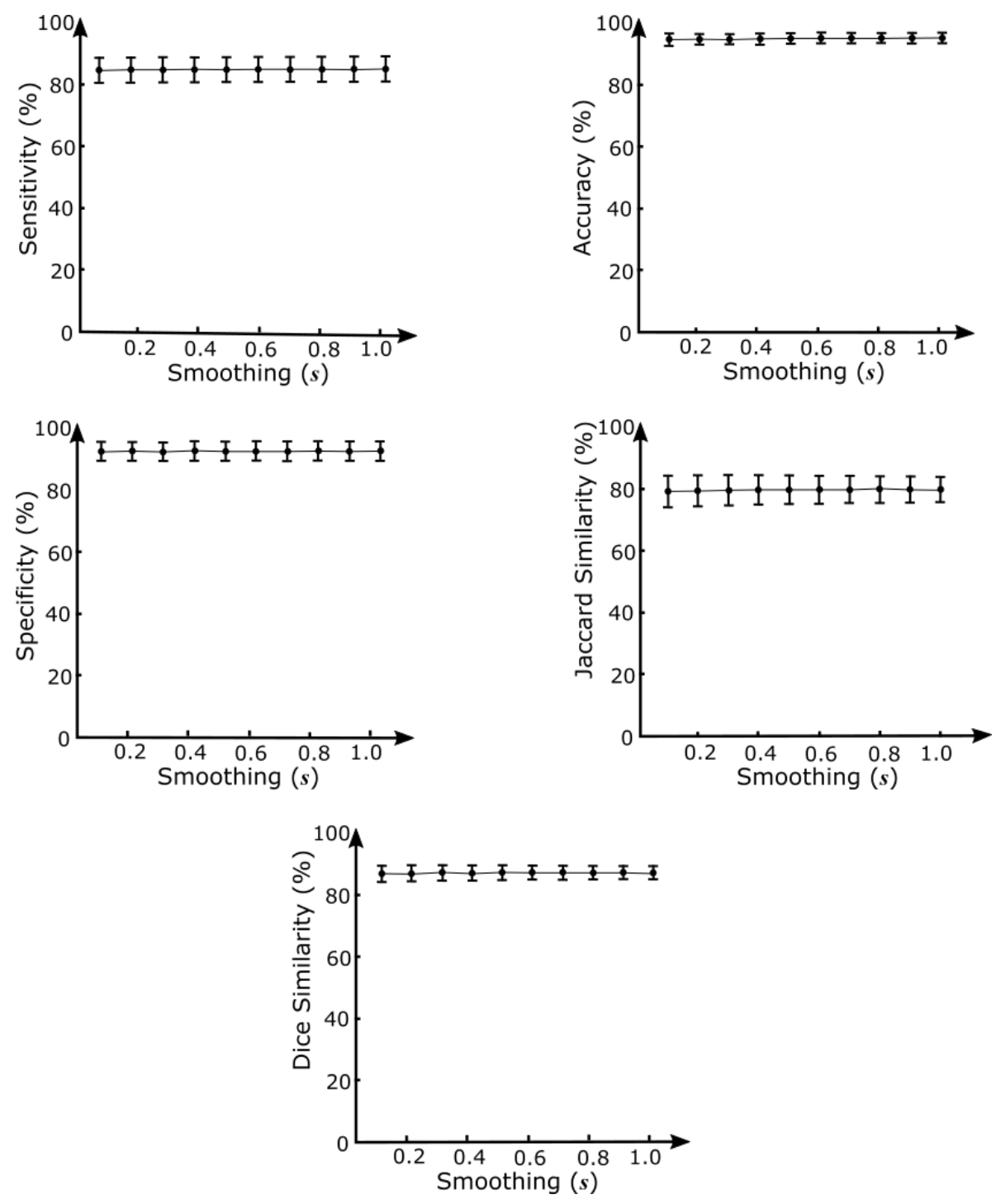

Figure 11: Performance variation of metrics $\ddot{J}, \ddot{D}$ (left graph), $\ddot{A}, \ddot{S}$ and $\bar{S}$ (right graph) with standard deviation using different values of smoothing $(s)$ for the QIN dataset. Note that small variations on performance indicate consistency across different values of $s$.

\section{Discussion}

In this section, we present some examples of over-estimated and underestimated results and explain our hypotheses why the proposed method unable 
to estimate those boundaries correctly. In addition, we will also present some segmentation results using a single active contour model, discuss the computational efficiency, compare the performance qualitatively of the proposed method with the existing methods in the literature, identify study limitations and discuss future directions.

\subsection{Over-estimated and Under-estimated Results}

Figure 12 shows examples with over-estimated and under-estimated pectoral boundaries. From our qualitative analysis, we found that it is harder for the proposed method if the breast area is small (e.g. first row) or the pectoral muscle is located too low in the image (e.g. second row). Our explanation for the first behaviour is that when the breast region is too small this affects the placement of $v_{1}$ and $v_{2}$ (hence, $v_{2}$ is mostly placed in the area of the pectoral muscle or chest wall). On the other hand, when the pectoral muscle is located too low in the contour $y_{L}^{0}$ the algorithm is likely to fail to capture it because this contour uses an inflation force and hence tends to evolve upwards. As a result, the boundary is under-estimated as shown in the second row of Figure 12 .
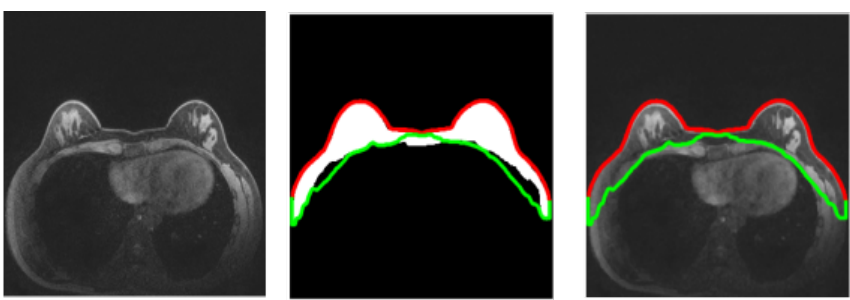

Jaccard $=75.4$

Dice $=86.1$

Sensitivity $=78.9$

Accuracy $=97.1$

Specificity $=99.4$

Hausdorff Distance $=7.1$
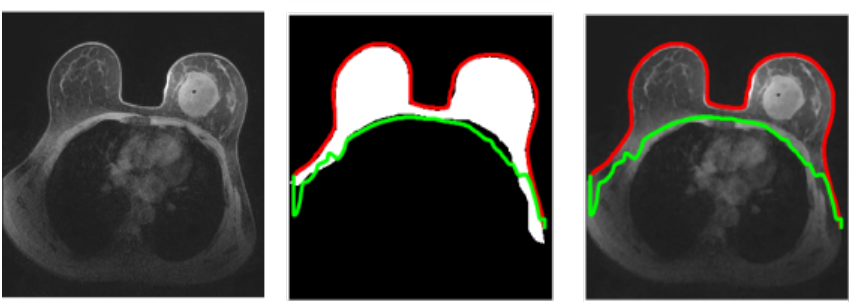

Jaccard $=78.4$

Dice $=87.5$

Sensitivity $=83.1$

Accuracy $=95.6$

Specificity $=98.5$

Hausdorff Distance $=8.7$

Figure 12: Example of segmentation results for breast (red) and pectoral muscle (green) boundary estimation with $\ddot{J}<80 \%$ taken from the QIN dataset. 

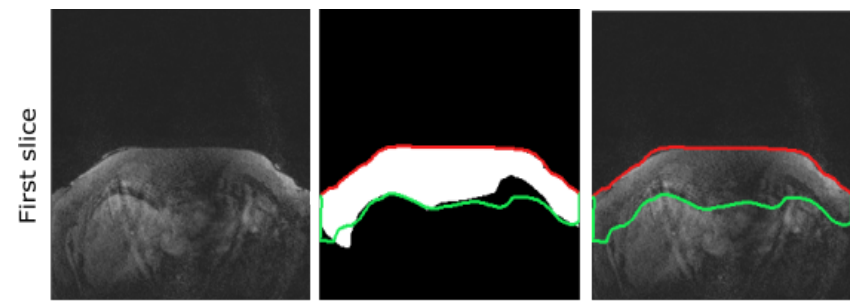

Jaccard $=71.5$

Dice $=80.8$

Sensitivity $=73.9$

Accuracy $=90.1$

Specificity $=93.2$

Hausdorff Distance $=9.1$
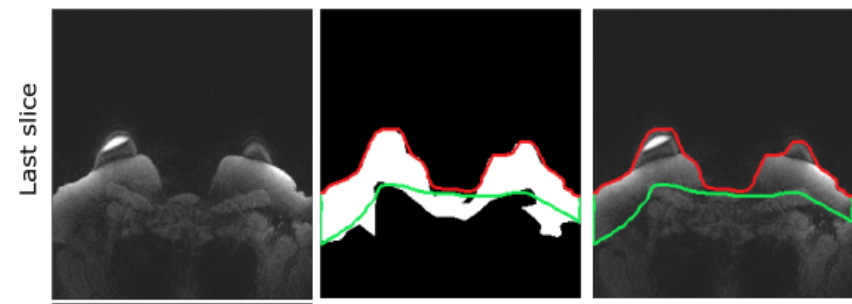

Jaccard $=68.4$

Dice $=77.8$

Sensitivity $=76.7$

Accuracy $=87.2$

Specificity $=91.3$

Hausdorff Distance $=10.2$
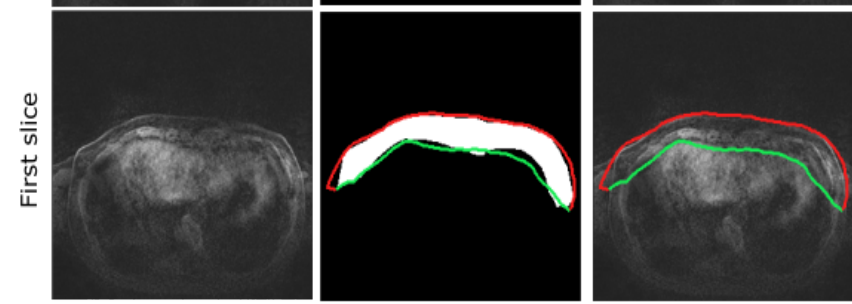

Jaccard $=78.3$

Dice $=88.9$

Sensitivity $=83.5$

Accuracy $=97.5$

Specificity $=99.5$

Hausdorff Distance $=6.4$
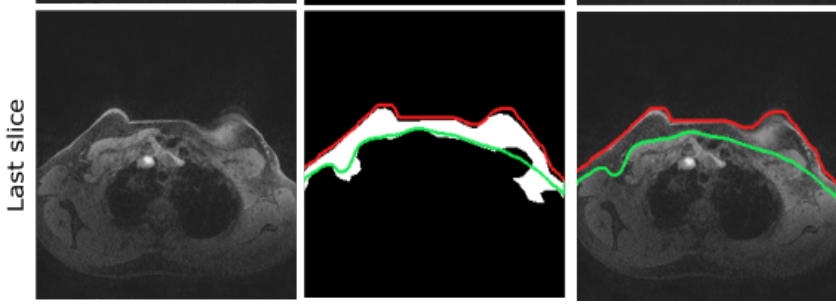

Jaccard $=72.6$

Dice $=80.5$

Sensitivity $=73.8$

Accuracy $=90.2$

Specificity $=93.8$

Hausdorff Distance $=8.9$

Figure 13: Example of segmentation results for breast (red) and pectoral muscle (green) boundary at the first and last slices taken from the QIN dataset.

Figure 13 shows examples of segmentation results at the first and last slices. It can be observed that the proposed method is unable to find the chest wall accurately due to invisible appearance of the boundary. However, since the subsequent direction of our study is to perform region/pixels classification within the breast region to find malignant regions; precise segmentation on these slices are less important because most malignant regions are visible within the middle slices. In addition, radiologists often use middle slices when assessing MR/CT scans rather than the first and last slices. The main reason for this is because 
most of the anatomical landmarks are more visible within the middle slices whereas the first and last slices usually do not contain malignant regions (or anatomical landmarks are obscured).

\subsection{Segmentation Results using a Single Active Contour}

In this section, we present segmentation results using an active contour to estimate both breast and chest wall boundaries simultaneously as well as using a single active contour to find the breast and chest wall separately. Note that the parameter values of $b=1.0$ and $s=1.0$ are the same for all experiments. Based on our experimental results and experience, using a single active contour usually fails to estimate both boundaries (or one of those boundaries) due to:

1. Noise can appear in the air-background (the upper side of the image in Figure 14 which can significantly affect the contour evolution if it starts from the the upper images moving towards the breast boundary. Hence, the contour evolution may need a larger number of iteration to find the actual boundary (which reduces the computational efficiency of the method). We show an example of this case in Figure 14 where the active contour needs 400 iterations before it can find the actual breast boundary (approximately 6 seconds) whereas our model took less than a second.

2. Strong appearance of other anatomical regions such as the liver, heart and lung can also influence contour evolution when finding the chest wall boundary as it tends to spread to that organ's region. We show an example in Figure 15 where the contour starts from the mask region (very close from the chest wall) and from $i=50$ to $i=200$ the contour evolution tends to spread to the other region.

3. A single active contour can't always track both boundaries at once because the chest wall boundary sometimes obscure or attach with the lung/heart/liver. We show an example in Figure 16 where the chest wall is obscure and has similar intensity/appearance with the heart and liver. First, we feed the active contour using Mask 1 (lower boundary is close to 


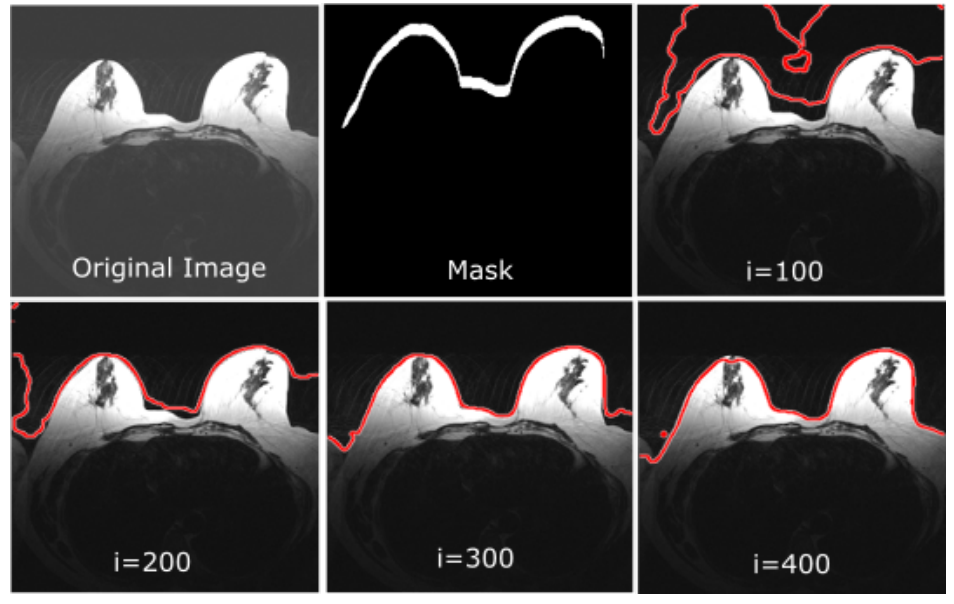

Figure 14: Contour evolution using different number of iterations on a noisy image.
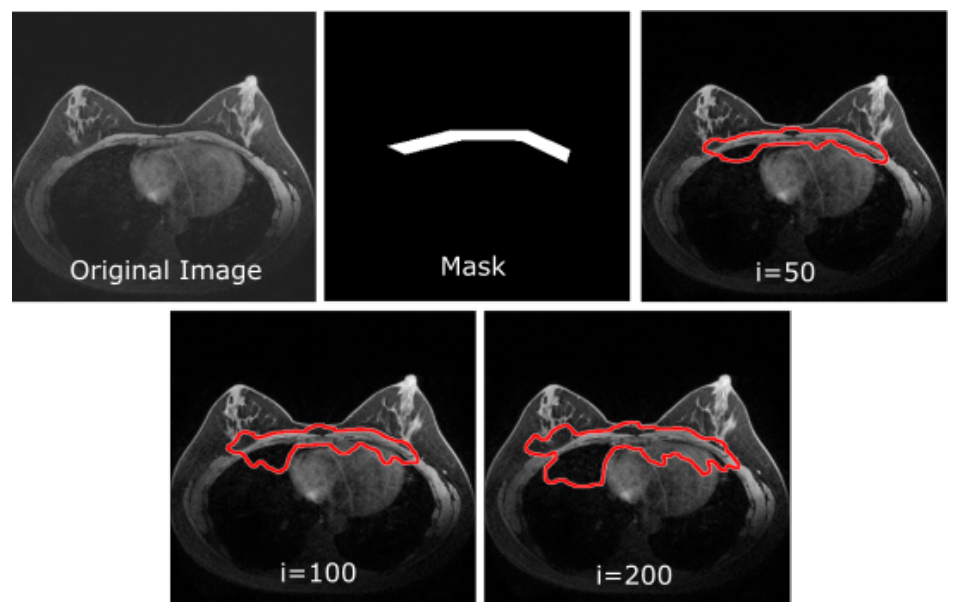

Figure 15: Contour evolution using different number of iterations on an image with strong appearance of the lung, liver and heart regions.

the chest wall boundary but away from the breast boundary) and second we feed the active contour using Mask 2 (where the upper boundary is located very close from the breast boundary but the masks base is away from the chest wall boundary). It can be observed that the contour evolution fails to find the chest wall and breast boundaries accurately and segmented the other parts of the image (liver/heart) in both experiments. 
In comparison, our proposed method can find the chest wall boundary

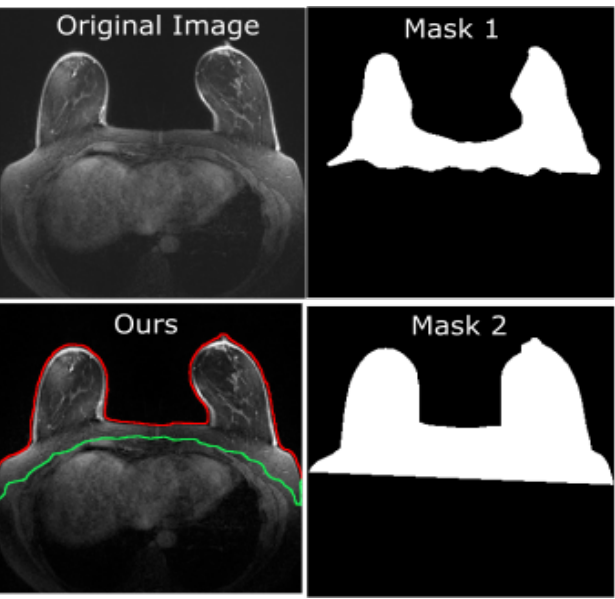

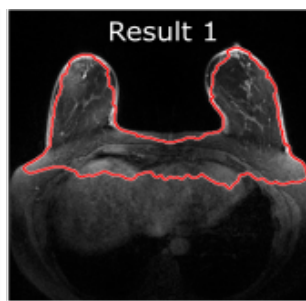

Result 2

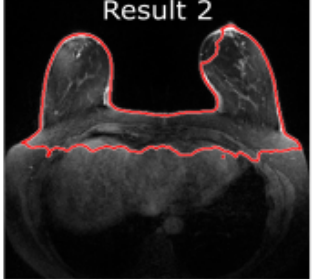

Figure 16: Using a single active contour to estimate both breast and chest wall boundaries simultaneously.

4. Invisible/complex anatomical appearance in the first-few and last-few slices (see Figure 13) which make active contour unable to find the right edges to approach and similar intensity homogeneity between the regions in the image also can affect the active contour movement/evolution (most breast and chest wall boundaries are visible in the middle slices). In this case (see Figure 13), a single active contour tends to over-segment the breast region due to invisible/weak edges along the boundary.

5. Using a single active contour to segment both boundaries simultaneously mean it is essential to get an initial mask that is close to these two boundaries. Unfortunately, to automatically get such mask is very difficult especially the bottom part of the breast where other organs appear (lung, liver and heart). In our case, we solved this problem using a generalised 2D mathematical model (the model of the breast shape) which is fast and can automatically estimate the initial boundary of the chest wall by generating two quadratic equations which eventually evolve using the GACM method. 
Therefore, in our study to estimate the chest wall boundary we developed a method consists of two separate sub-methods that firstly estimates the breast boundary followed by contours evolution. The initial contours were determined automatically based on the generalised 2D breast model and were evolved by exploiting the inflation and deflation forces of the GACM.

\subsection{Computational Efficiency}

In terms of computational efficiency the proposed method took on average $4.13 \pm 1.3$ seconds to find both breast and pectoral muscle boundaries with the following parameter settings: $i=100, s=1.0, d=10, b=-1$ for $y_{U}^{0}$ and $b=1$ for $y_{L}^{0}$. This is including the pre-processing and post-processing steps. The GACM itself took on average $3.1 \pm 1.2$ seconds to find the pectoral muscle boundary. Employing our proposed method is easy in terms of the choice of the parameters due to its consistency over a wide range of different parameter values. For example, the choice of parameters $i$ in the range $[70, \ldots, 100], d$ in the range $[5, \ldots, 10], b$ in the range $[0.6,1.0]([-1.0(-0.6)])$ and $s$ in the range $[0.4, \ldots, 1.0]$ should produce a consistent performance based on our experimental results.

\subsection{Qualitative Comparison}

${ }_{630}$ Quantitative comparison of different studies in the literature is very difficult due to the unavailability of data from the other authors, different evaluation metrics and different numbers of images used. Table 2 shows qualitative comparison with the existing studies in the literature. Note that each study has its own dataset which makes a direct comparison difficult. Nevertheless, for the sake of comparison, the proposed method achieved up to $\ddot{J}=81.1 \%, \ddot{D}=89.4 \%$, $\ddot{A}=96.1 \%, \ddot{S}=88.1 \%, \bar{S}=98.4 \%$ and $\nabla D=5.71 \mathrm{~mm}$ when tested on the QIN dataset. We further evaluate the proposed method on our own dataset and it achieved $\ddot{J}=84.9 \%, \ddot{D}=92.3 \%, \ddot{A}=97.8 \%, \ddot{S}=90.2 \%, \bar{S}=98.8 \%$ and $\nabla D=4.92 \mathrm{~mm}$ based on 50 patients. The method of Thakran et al. [7] reported ${ }_{640}$ volumetric evaluation of $\ddot{J}=95.1 \%$ and $\ddot{D}=97.7$ based on 30 patients. The 
methods of Fooladivanda et al. 9], Milenković et al. [8, Jiang et al. (2017) [11] and Wu et al. 10] produced very similar results between $\ddot{D}=95$ to $96.4 \%$. Note that studies in 9, 11] have made volumetric evaluation whereas the majority of the studies in the literature made $2 \mathrm{D}$ evaluation.

Table 2: Qualitative comparison with the existing studies.

\begin{tabular}{|c|c|c|c|c|}
\hline Authors & $\ddot{J}(\%)$ & $\ddot{D}(\%)$ & \# Patients & Evaluation \\
\hline \multirow[t]{2}{*}{ Proposed } & 81.1 & 89.4 & 9 & 2D (QIN dataset) \\
\hline & 84.9 & 92.3 & 50 & 2D (Own dataset) \\
\hline Thakran et al. (2018) [7] & 95.1 & 97.7 & 30 & $3 \mathrm{D}$ \\
\hline Fooladivanda et al. (2017) 9] & - & 96.4 & 42 & $2 \mathrm{D}$ \\
\hline Milenković et al. (2015) 8 & - & 96.1 & 52 & $2 \mathrm{D}$ \\
\hline Jiang et al. (2017) 11 & - & 95.8 & 100 & $3 \mathrm{D}$ \\
\hline Wu et al. (2013) 10 & - & 95 & 60 & $2 \mathrm{D}$ \\
\hline Gubern-Mérida (2015) 5 & - & 94 & 27 & $2 \mathrm{D}$ \\
\hline Rosado-Toro et al. (2015) 6] & - & 92 & 14 & $2 \mathrm{D}$ \\
\hline Giannini et al. (2010) 4 & - & 79 & 31 & $2 \mathrm{D}$ \\
\hline
\end{tabular}

Giannini et al. 4] reported $\ddot{S}=79 \% \pm 9 \%, \ddot{P}=82 \% \pm 10 \%$ and $\ddot{R}=$ $95 \% \pm 2 \%$ based on 31 female patients, whereas our proposed method achieved $\ddot{P}=94 \% \pm 7.2 \%$ and $\ddot{R}=87.1 \% \pm 6.2 \%$. The metric $\ddot{P}$ is the number of pixels in the intersection of segmentation and reference (manual segmentation), divided by the number of pixels in the segmentation alone. The metric $\ddot{R}$ measures the ratio of the number of pixels in the intersection of segmentation and reference, to the number of pixels in the reference. The study of Gubern-Mérida et al. [5] reported $\ddot{D}=94 \% \pm 3 \%$ and $\ddot{S}=96 \% \pm 2 \%$ based on 27 female patients. From the results of the existing studies presented in 2 , we achieved comparable performance considering the size of dataset used in our study (evaluated on 59 patients). In terms of ground truth, our manual segmentation on each slice was provided by an experienced observer (QIN dataset) and an expert radiologist 
(our own dataset). This is similar to the studies in [5, 9, 10] where a single experienced observer/an expert radiologist was used to provide the manual annotation. Although the other studies used two observers/radiologists to provide their annotation, each slice/scan/patient was annotated by one radiologist only (another radiologist provides annotation for another patient) such as the studies in [4, 6]. This means, using two radiologists are to speed up the process of providing ground truth because it is time consuming.

\subsection{Study limitations}

The main limitations of this study is that we are unable to compare our results quantitatively against the other methods in the literature due to the absence of data with ground truth or manual annotation available publicly. To address this issue, we plan to make our ground truth data available so that future researchers can compare their results with our results quantitatively. Furthermore, implementing the existing studies in the literature from scratch is time consuming and the risk of developing/implementing it incorrectly is high. Another limitation of our study is no quantitative reproducibility has been established/provided for the QIN dataset. It is extremely time consuming for our radiologist to provide his own annotations and make quantitative comparison with our own annotation.

\subsection{Future work}

For future work we plan to improve the contours' convergence, hence boosting the overall performance of the proposed method. We plan to employ a robust segmentation technique to improve the accuracy of breast boundary. The estimated breast boundary influences most of the important landmarks in the $2 \mathrm{D}$ model such as $p_{3}, p_{4}, p_{5}, p_{6}, v_{1}$ and $v_{2}$. For example, a poor estimation of breast boundary resulting poor location of these points in the $2 \mathrm{D}$ model, hence poor initialisation of $y_{U}^{0}$ and $y_{L}^{0}$. Therefore, it is necessary to have a more robust method to estimate the breast boundary. However, achieving an optimum 


\section{Conclusions}

We have developed a new breast segmentation method for MR images using a generalised 2D mathematical breast model in conjunction with the geodesic active contour model (GACM). The 2D breast model was developed based on an empirical and quantitative evaluation to define a general shape (axial plane) of

\section{Acknowledgment}

This research was undertaken as part of the Decision Support and Information Management System for Breast Cancer (DESIREE) project. The project has received funding from the European Union's Horizon 2020 research and innovation programme under grant agreement No 690238. 
[1] Cancer Research UK, Breast cancer statistics (2014).

a URL http://www.cancerresearchuk.org/health-professional/

cancer-statistics/statistics-by-cancer-type/breast-cancer\# heading-Zero

[2] Cancer Research UK, Breast cancer statistics (2014).

n [3] L. Wang, K. Filippatos, O. Friman, H. K. Hahn, Fully automated segmentation of the pectoralis muscle boundary in breast mr images, Vol. 7963, 2011, pp. 7963-7963-8. doi:10.1117/12.877645.

URL http://dx.doi.org/10.1117/12.877645

[4] V. Giannini, A. Vignati, L. Morra, D. Persano, D. Brizzi, L. Carbonaro, A. Bert, F. Sardanelli, D. Regge, A fully automatic algorithm for segmentation of the breasts in dce-mr images, in: 2010 Annual International Conference of the IEEE Engineering in Medicine and Biology, 2010, pp. 3146-3149.

[5] A. Gubern-Mérida, M. Kallenberg, R. M. Mann, R. Martí, N. Karssemeijer, Breast segmentation and density estimation in breast mri. a fully automatic framework., IEEE Trans. Biomedical and Health Informatics 19 (1) (2015) 349-357.

[6] J. A. Rosado-Toro, T. Barr, J.-P. Galons, M. T. Marron, A. Stopeck, C. Thomson, P. Thomson, D. Carroll, E. Wolf, M. Altbach, J. J. Rodríguez, Automated breast segmentation of fat and water $\mathrm{mr}$ images using dynamic programming, Academic Radiology 22 (2) (2015) 139-148.

口 [7] S. Thakran, S. Chatterjee, M. Singhal, A. S. R. K. Gupta, Automatic outer ם and inner breast tissue segmentation using multi-parametric mri images of 
breast tumor patients, PLos ONE 13 (1) (2018) e0190348.

URL .https://doi.org/10.1371/journal .pone.0190348

[14] C. Gnonnou, N. Smaoui, Segmentation and 3d reconstruction of mri images for breast cancer detection, in: International Image Processing, Applicaregion segmentation in the axial breast mr images, Computers in Biology and Medicine 62 (2015) 55-64.

[9] A. Fooladivanda, S. B. Shokouhi, N. Ahmadinejad, Breast-region segmentation in mri using chest region atlas and svm, Turkish Journal of Electrical Engineering \& Computer Sciences 25 (2017) 4575-4592.

[10] S. Wu, S. P. Weinstein, E. F. Conant, M. D. Schnall, D. Kontos, Automated chest wall line detection for whole-breast segmentation in sagittal breast mr images, Med. Phys. 40 (4) (2013) 1-12.

[11] L. Jiang, X. Hu, Q. Xiao, Y. Gu, Q. Li, Fully automated segmentation of whole breast using dynamic programming in dynamic contrast enhanced mr images, Med. Phys. 44 (6) (2017) 2400-2414.

[12] A. Yoo, K. W. Minn, U. S. Jin, Magnetic resonance imaging-based volumetric analysis and its relationship to actual breast weight, Archives of Plastic Surgery 40 (2013) 203-208.

[13] D. J. Thompson, M. O. Leach, S. A. G. G. Kwan-Lim, S. J. Ramus, I. Warsi, F. Lennard, M. Khazen, E. Bryant, S. Reed, C. R. M. Boggis, D. G. Evans, R. A. Eeles, D. F. Easton, R. M. L. Warren, T. U. study of MRI screening for breast cancer in women at high risk (MARIBS), Assessing the usefulness of a novel mri-based breast density estimation algorithm in a cohort of women at high genetic risk of breast cancer: the UK MARIBS study, Breast Cancer Research 11 (2009) R80.

tions and Systems Conference, 2014, pp. 1-6. doi:10.1109/IPAS.2014.

[8] J. Milenković, O. Chambers, M. M. Mušič, J. F. Tasič, Automated breast7043316 . 
[15] J. S. et al., The mammographic image analysis society digital mammogram database, in: Proc. Excerpta Med. Int. Congr. Ser., 1994, pp. 375-378.

[16] I. C. Moreira, I. Amaral, I. Domingues, A. Cardoso, M. J. Cardoso, J. S. Cardoso, Inbreast: toward a full-field digital mammographic database, Acad Radiol. 19 (2) (2011) 236-428.

[17] M. A. G. Lopez, N. G. de Posada, D. C. Moura, R. R. Pollan, J. M. F. Valiente, C. S. Ortega, M. R. del Solar, G. D. Herrero, I. M. A. P. Ramos, J. P. Loureiro, T. C. Fernandes, B. M. F. de Araújo, Bcdr: A breast cancer digital repository, in: ICEM15: 15th International Conference on Experimental Mechanics, FEUP-EURASEM-APAET, 2012, pp. 1-5.

[18] M. Heath, K. Bowyer, D. Kopans, R. Moore, W. P. Kegelmeyer, The digital database for screening mammography, in: Proceedings of the Fifth International Workshop on Digital Mammography, Medical Physics Publishing, 2001, pp. 212-218.

[19] G. Ertaş, H. O. Gülür, O. Osman, O. N. Uşan, M. Tunaci, M. Dursu, Breast MR segmentation and lesion detection with cellular neural networks and 3d template matching, Comp. Biol.and Med. 38 (1) (2008) 116-126.

[20] T. Twellmann, O. Lichte, T. W. Nattkemper, An adaptive tissue characterization network for model-free visualization of dynamic contrast-enhanced magnetic resonance image data, IEEE Transactions on Medical Imaging 24 (10) (2005) 1256-1266. doi:10.1109/TMI .2005.854517.

[21] T. Lee, Active contour models, Master's thesis, Oregon State University, the United States (2005).

[22] M. Kass, A. Witkin, D. Terzopoulos, Snakes: Active contour models, Int. J. Comput. Vision 55 (7) (1992) 14-26.

[23] M. Ciecholewski, An edge-based active contour model using an inflation/deflation force with a damping coefficient, Expert Systems with Applications 44 (2016) 22-36. 
[24] L. Álvarez, L. Baumela, P. Henríquez, P. Márquez-Neila, Morphological

[25] M. A. Wirth, A. Stapinski, Segmentation of the breast region in mammograms using snakes, in: First Canadian Conference on Computer and Robot

[27] J. Miller, D. Breen, W. Lorensen, R. O'Bara, M. Wozny, Geometrically snakes, in: 2010 IEEE Computer Society Conference on Computer Vision and Pattern Recognition, 2010, pp. 2197-2202. doi:10.1109/CVPR.2010. 5539900 .

Vision, 2004. Proceedings., 2004, pp. 385-392. doi:10.1109/CCCRV. 2004. 1301473

[26] V. Miller, D. Breen, M. Wozny, Extracting geometric models through constraint minimization, in: Proceedings of the 1st Conference on Visualization '90, VIS '90, IEEE Computer Society Press, Los Alamitos, CA, USA, 1990, pp. $74-82$.

URL http://dl.acm.org/citation.cfm?id=949531.949543

deformed models: A method for extracting closed geometric models form volume data, SIGGRAPH Comput. Graph. 25 (4) (1991) 217-226. doi: 10.1145/127719.122742.

URL http://doi.acm.org/10.1145/127719.122742

[28] T. F. Chan, L. A. Vese, Active contours without edges, IEEE Transactions on Image Processing 10 (2) (2001) 266-277. doi:10.1109/83.902291.

[29] C. Li, R. Huang, Z. Ding, J. C. Gatenby, D. N. Metaxas, J. C. Gore, A level set method for image segmentation in the presence of intensity inhomogeneities with application to mri, IEEE Transactions on Image Processing 20 (7) (2011) 2007-2016. doi:10.1109/TIP.2011.2146190.

[30] K. Zhang, H. Song, L. Zhang, Active contours driven by local image fitting energy, Pattern Recognition 43 (4) (2010) 1199-1206.

[31] A. Belaid, D. Boukerroui, Y. Maingourd, J. Lerallut, Phase based level set 
segmentation of ultrasound images, in: 2009 9th International Conference on Information Technology and Applications in Biomedicine, 2009, pp. 1-4.

[32] A. Khadidos, V. Sanchez, C.-T. Li, Weighted level set evolution based on local edge features for medical image segmentation, IEEE Transactions on Image Processing 26 (4) (2017) 69-82.

[33] H. S. K. Zhang, L. Zhang, D. Zhang, Reinitialization-free level set evolution via reaction-diffusion, IEEE Transactions on Image Processing 22 (1) (2013) 258-271.

[34] V. Estellers, D. Zosso, X. Bresson, J.-P. Thiran, Harmonic active contours, IEEE Transactions on Image Processing 23 (1) (2014) 69-82.

[35] V. Caselles, R. Kimmel, G. Sapiro, Geodesic active contours, International Journal of Computer Vision 22 (1) (1997) 61-79.

[36] W. C. Y.-l. C. Y. L. L.-W. T. Y. Zhou, W.-R. Shi, D.-Q. Chen, Active contours driven by localizing region and edge-based intensity fitting energy with application to segmentation of the left ventricle in cardiac ct images, Neurocomputing 156 (2015) 199-210.

[37] K. Zuiderveld, Contrast limited adaptive histograph equalization, in: Proceedings of the Graphic Gems IV, San Diego: Academic Press Professional, 1994, pp. 474-485.

${ }_{840}[38]$ N. Otsu, A threshold selection method from gray-level histograms, IEEE

【 Transactions on Systems, Man, and Cybernetics 9 (1) (1979) 62-66. doi: 10.1109/TSMC.1979.4310076.

[39] M. Kass, A. Witkin, D. Terzopoulos, Snakes: Active contour models,

口 International Journal of Computer Vision 1 (4) (1988) 321-331. doi:

$845 \quad 10.1007 / \mathrm{BF} 00133570$

URL https://doi.org/10.1007/BF00133570 
[40] N. Paragios, R. Deriche, Geodesic active contours and level sets for the detection and tracking of moving objects, IEEE Transactions on Pattern

ロ Analysis and Machine Intelligence 22 (3) (2000) 266-280. doi:10.1109/ 34.841758 .

[41] National biomedical imaging archive (nbia).

URL https://imaging.nci.nih.gov/ncia/login.jsf

[42] W. Huang, X. Li, Y. Chen, X. Li, M. C. Chang, M. J. Oborski, D. I. Malyarenko, M. Muzi, G. H. Jajamovich, A. Fedorov, A. Tudorica, S. N. Gupta, C. M. Laymon, K. I. Marro, H. A. Dyvorne, J. V. Miller, D. P. Barbodiak, T. L. Chenevert, T. E. Yankeelov, J. M. Mountz, P. E. Kinahan, R. Kikinis, B. Taouli, F. Fennessy, J. Kalpathy-Cramer, The cancer imag-

a ing archive: Variations of dynamic contrast-enhanced magnetic resonance

i. imaging in evaluation of breast cancer therapy response: a multicenter data analysis challenge (2014).

URL http://doi.org/10.7937/K9/TCIA.2014.A2N1IXOX

[43] Wikipedia, Jaccard index.

URL https://en.wikipedia.org/wiki/Jaccard_index

[44] Wikipedia, Sørensendice coefficient,

865

URL https://en.wikipedia.org/wiki/S\%C3\%B8rensen\%E2\%80\%93Dice_ coefficient

[45] Wikipedia, Hausdorff distance.

URL https://en.wikipedia.org/wiki/Hausdorff_distance 